\title{
A MODAL ANALYSIS OF \\ LAMEILAR DIFFRACTION GRATINGS IN CONICAL MOUNTINGS
}

\author{
Lifeng $\mathrm{Li}$ \\ Optical Sciences Center \\ University of Arizona \\ Tucson, AZ, 85721, U.S.A.
}

\begin{abstract}
A rigorous modal analysis of lamellar gratings, i.e., gratings having rectangular grooves, in conical mountings is presented. It is an extension of the analysis of Botten et al which considered non-conical mountings. A key step in the extension is a decomposition of the electromagnetic field in the grating region into two orthogonal components. A computer program implementing this extended modal analysis is capable of dealing with plane wave diffraction by dielectric and metallic gratings with deep grooves, at arbitrary angles of incidence, and having arbitrary incident polarizations. Some numerical examples are included.
\end{abstract}

\section{INTRODUCTION}

The modal approach has been applied by many authors to lamellar, non-perfectly conducting gratings in the past [1-10]. Most noticeably, Botten et al. [5-7] presented a series of three papers, in 1981, on the modal analysis of dielectric, finitely conducting, and highly 
conducting lamellar gratings. Their work was later formulated in a more systematic way, and its certain numerical aspects were improved, by Suratteau et al. [8] and by Tayeb and Petit [9]. However, these analyses are limited to non-conical mountings. In many applications, lamellar gratings are used in conical mountings [11]. Recently, a modal analysis of lamellar gratings in conical mountings was presented by Peng [12].

The present work differs substantially from that of Peng [12] in mathematical formulation and numerical implementation. In Peng's work, the validity of the decomposition of the electromagnetic field in the corrugated region into two orthogonal components is assumed without proof. The eigen-functions (the modal fields in the corrugated region) for a grating in a conical mountings are constructed by geometrical means from the TE and TM eigen-functions for the grating in a equivalent non-conical (also called classical) mounting. The completeness and orthogonality of the eigen-functions assembled in this manner are not addressed. In this paper, the eigen-functions along with their completeness and orthogonality are derived rigorously and systematically from the boundaryvalue problems.

The present work can be considered as an extension of the works of Botten et al. and Suratteau et al. to conical mountings. A key step in its development is the proof of the field decomposition mentioned above. Once this is done, the task of finding eigenvalues and eigen-functions for a conical mounting reduces to that of a classical mounting, and the previous results of the above authors, including their powerful and sophisticated numerical 
methods for finding the eigenvalues, can be used.

The mathematical formulation of the modal analysis is presented in section 2 , and the numerical aspects of the analysis are addressed in section 3. Section 4 provides some numerical results. The validity of the field decomposition is proved in Appendix A. For the sake of the normal flow of the paper, some of the results available in Ref. 5-9 are re-stated in sections $\mathbf{2}$ and 3, but often are formulated differently. In other instances, the reader is referred to the original references.

\section{MATHEMATICAL FORMULATION}

\subsection{Notation}

A lamellar grating in a conical diffraction configuration is depicted in Fig. 1. The coordinate system is chosen such that the $x$-axis is perpendicular to, and the $z$-axis is parallel to, the grating grooves, and the $y$-axis is the normal of the overall structure. A monochromatic plane wave of vacuum wavelength $\lambda_{0}$ is incident on the lamellar grating at a polar angle $\theta$ and an azimuthal angle $\phi$. The range of $\theta$ is $0 \leq \theta<\pi / 2$ and that of $\phi$ is $-\pi<\phi \leq \pi$, with the clockwise direction being the positive direction for $\phi$. The same conventions will be used for the diffracted waves that are not shown. The incident polarization is in general elliptical.

The geometry of the lamellar grating is shown in Fig. 2. The grating period is $d$, and the widths of medium 1 and medium 2 are $d_{1}$ and $d_{2}$. We shall call the regions of space 
where $y>h / 2, y<-h / 2$, and $-h / 2<y<h / 2$, regions 1,2 , and 0 , respectively, where $h$ is the grating groove depth. We shall use the superscript $(j)$, where $j=1,2$, to denote quantities associated with regions $j$ and the subscript $j$ to denote quantities associated with the two media in region 0 . Thus, the permittivity and permeability of the medium in region 1 are $\epsilon^{(1)}$ and $\mu^{(1)}$, and those in region 2 are $\epsilon^{(2)}$ and $\mu^{(2)}$. The permittivity and permeability of region 0 are periodic functions of $x$,

$$
\left.\begin{array}{rrr}
\epsilon(x)=\epsilon_{1}, & \mu(x)=\mu_{1}, & 0 \leq|x| \leq d_{1} / 2, \\
\epsilon(x)=\epsilon_{2}, & \mu(x)=\mu_{2}, & d_{1} / 2<|x| \leq d / 2 .
\end{array}\right\}
$$

Although for most optical applications, the permeability is a constant and equals that of the vacuum, to reveal the symmetry of the electric and magnetic fields, $\mu_{1}$ and $\mu_{2}$ are formally assumed to be different. The Gaussian system of units is used in this paper.

Let $k_{0}$ be the magnitude of the vacuum wave-vector. The magnitudes of the wavevector in regions $j=1,2$ and region 0 are denoted by $k^{(j)}$ and $k(x)$,

$$
\begin{aligned}
k^{(j) 2} & =\epsilon^{(j)} \mu^{(n)} k_{0}^{2}, \\
k^{2}(x) & =\epsilon(x) \mu(x) k_{0}^{2} .
\end{aligned}
$$

The wave-vector of the incident plane wave is

$$
E=k^{(1)}(\hat{x} \sin \theta \cos \phi-\hat{y} \cos \theta+z \sin \theta \sin \phi) .
$$

We denote the $z$-component of the incident wave-vector by $k_{z}$

$$
k_{z}=k^{(1)} \sin \theta \sin \phi \text {. }
$$

and we define the reduced magnitudes of the wave-vectors by $k^{())}$and $\bar{k}(x)$, 


$$
\begin{aligned}
& k^{(j) 2}=k^{(j) 2}-k_{z}^{2}, \\
& k^{2}(x)=k^{2}(x)-k_{z}^{2} .
\end{aligned}
$$

Since the physical problem is time and $z$-invariant, the electromagnetic field may be assumed to be of the form

$$
\begin{aligned}
& E(x, y, z, t)=\vec{E}(x, y) e^{i k_{z} z-i \omega t}, \\
& H(x, y, z, t)=\vec{H}(x, y) e^{i k_{z}-t \omega t} .
\end{aligned}
$$

Throughout this paper, $i=\jmath / 1$. Substituting (8a) and (8b) into Maxwell's equations, we may express the transverse components of the electromagnetic field in terms of the longitudinal (z) components,

$$
\begin{aligned}
& \vec{E}_{t}(x, y)=\frac{i}{k^{2}(x)}\left[k_{z} \nabla_{t} E_{z}(x, y)-\mu k_{0} \hat{z} \times \nabla_{t} H_{z}(x, y)\right], \\
& \vec{H}_{z}(x, y)=\frac{i}{k^{2}(x)}\left[k_{z} \nabla_{t} H_{z}(x, y)+\epsilon k_{0} \hat{z} \times \nabla_{t} E_{z}(x, y)\right],
\end{aligned}
$$

where $\nabla_{t}=\hat{x} \partial_{x}+\varphi \partial_{y}$. So in solving the conical diffraction problem, it is only necessary to work with the $z$-components of the electric and magnetic fields.

As usual, the fields above and below the corrugated region may be written in Rayleigh expansions [13]:

$$
\begin{aligned}
& E_{z}(x, y)=I_{z}^{(0)} e^{\left\{\sigma_{0} x-1 \beta_{0}^{(1)} y\right.}+\sum_{n=-\infty}^{\infty} R_{n}^{(0)} e^{\left\{\alpha_{z} x+i \beta_{n}^{(0) y},\right.} \\
& H_{z}(x, y)=I_{z}^{(h)} e^{i \epsilon_{0} x-i \beta_{\theta}^{(n)} y}+\sum_{n=-\infty}^{+\infty} R_{n}^{(n)} e^{i \varepsilon_{n} x+i \beta_{x}^{(1)} y}
\end{aligned}
$$


for $y>h / 2$, and

$$
\begin{aligned}
& E_{z}(x, y)=\sum_{n=-\infty}^{+\infty} T_{n}^{(0)} e^{i \alpha_{n} x-i \beta_{n}^{\infty} y}, \\
& H_{z}(x, y)=\sum_{n=-\infty}^{+\infty} T_{n}^{(n)} e^{i \alpha_{n} x-i \beta_{a}^{(D)} y}
\end{aligned}
$$

for $y<h / 2$, where

$$
\begin{aligned}
& \alpha_{n}=\alpha_{0}+2 n \pi / d, \quad \alpha_{0}=k^{(1)} \sin \theta \cos \phi, \\
& \beta_{n}^{(j) 2}=\bar{k}^{(j) 2}-\alpha_{n}^{2}, \quad \operatorname{Re}\left[\beta_{n}^{(j)}\right]+\operatorname{Im}\left[\beta_{n}^{(j)}\right]>0 .
\end{aligned}
$$

In (10) and (11), $I_{z}^{(e)}, I_{z}^{(h)}, R_{n}^{(e)}, R_{n}^{(h)}, T_{n}^{(e)}$, and $T_{n}^{(h)}$ are, respectively, the complex amplitudes of the $z$-components of the incident and diffracted electric and magnetic fields in regions 1 and 2.

\subsection{Field Decomposition}

Suppose $h$ tends to infinity in Fig. 2, yielding a medium that is periodic in $x$ and infinite in $y$ and $z$. If the general expression for the electromagnetic field in this infinite medium is known, by imposing the interface conditions at $y= \pm h / 2$ between this field and those given in (10) and (11), we can determine all the unknown field amplitudes.

For a $z$ - and $y$-invariant medium, the following system of equations for the fields $E_{z}$ and $H_{z}$ can be derived from the original Maxwell's equations 


$$
\left(\begin{array}{ll}
\frac{k^{2}}{\epsilon} \frac{\partial}{\partial x}\left(\frac{\epsilon}{k^{2}} \frac{\partial}{\partial x}\right)+\frac{\partial^{2}}{\partial y^{2}}+\hbar^{2} & +\frac{k_{z}}{k_{0}} \frac{k^{2}}{\epsilon}\left(\frac{d}{d x} \frac{1}{\tilde{k}^{2}}\right) \frac{\partial}{\partial y} \\
-\frac{k_{z}}{k_{0}} \frac{\bar{k}^{2}}{\mu}\left(\frac{d}{d x} \frac{1}{\bar{k}^{2}}\right) \frac{\partial}{\partial y} & \frac{k^{2}}{\mu} \frac{\partial}{\partial x}\left(\frac{\mu}{\bar{k}^{2}} \frac{\partial}{\partial x}\right)+\frac{\partial^{2}}{\partial y^{2}}+\tilde{k}^{2}
\end{array}\right)=0 .
$$

To determine the modal representation of the electromagnetic field we need to solve (14) subject to the pseudo-periodic boundary conditions [13]

$$
\left.\begin{array}{lr}
E_{z}(d / 2, y)=e^{i \theta_{0} d} E_{z}(-d / 2, y), & H_{z}(d / 2, y)=e^{i \alpha_{0} d} H_{z}(-d / 2, y), \\
\frac{\partial E_{z}}{\partial x}(d / 2, y)=e^{i \alpha_{0} d} \frac{\partial E_{z}}{\partial x}(-d / 2, y), & \frac{\partial H_{z}}{\partial x}(d / 2, y)=e^{i \alpha_{0} d} \frac{\partial H_{z}}{\partial x}(-d / 2, y) .
\end{array}\right\}
$$

In addition, we also need the interface conditions for the fields and their derivatives at the medium discontinuities. However, the burden of mentioning the interface conditions can be relieved if we understand Maxwell's equations in the sense of distribution [13].

Equation (14) is a coupled system of equations for $E_{z}$ and $H_{z}$, whose direct solution seems to be difficult. However, by the following field decomposition, its solution is simplified. In a $z$ - and $y$-invariant medium, an electromagnetic field is said to be $E_{\perp}\left(H_{\perp}\right)$ if the $x$ component of its electric (magnetic) field vanishes. Let the superscript (e) denote the $E_{\perp}$ field, and $(h)$ the $H_{\perp}$ field. Then from (9)

$$
\begin{aligned}
& k_{z} \frac{\partial}{\partial x} E_{z}^{(s)}+\mu k_{0} \frac{\partial}{\partial y} H_{z}^{(s)}=0, \\
& k_{z} \frac{\partial}{\partial x} H_{z}^{(k)}-\epsilon k_{0} \frac{\partial}{\partial y} E_{z}^{(h)}=0 .
\end{aligned}
$$

In Appendix $\mathrm{A}$, we prove that any field $\left(E_{z}, H_{z}\right)^{\mathrm{T}}$, where superscript $\mathrm{T}$ signifies matrix transpose, satisfying (14) and (15) can be uniquely expressed as a sum of an $E_{\perp}$ field and 
an $\mathrm{H}_{\perp}$ field, and these two component fields satisfy (14) and (15) independently. Thus the task of solving the vector-valued boundary-value problem (14) plus (15) is reduced to two independent scalar ones to be derived below.

Using (16a) to eliminate $H_{z}$ from the first equation of (14), and using (16b) to eliminate $E_{z}$ in the second of (14), we obtain,

$$
\begin{aligned}
& \mu \frac{\partial}{\partial x}\left(\frac{1}{\mu} \frac{\partial}{\partial x} E_{z}^{(0)}\right)+\frac{\partial^{2}}{\partial y^{2}} E_{z}^{(0)}+k^{2} E_{z}^{(0)}=0, \\
& \epsilon \frac{\partial}{\partial x}\left(\frac{1}{\epsilon} \frac{\partial}{\partial x} H_{z}^{(1)}\right)+\frac{\partial^{2}}{\partial y^{2}} H_{z}^{(1)}+k^{2} H_{z}^{(1)}=0 .
\end{aligned}
$$

In these two equations, the electric field and the magnetic field are no longer coupled. Actually, these two equations are identical to the equations for the TE and TM polarized fields in classical mountings [5-8], provided that $k^{2}$ is replaced by $\hat{k}^{2}$.

The symmetry exhibited by (17a) and (17b) with respect to $E_{z}^{(e)}$ and $H_{z}^{(h)}$ and with respect to $\epsilon$ and $\mu$ suggests that they can be rewritten as

$$
\sigma^{(s)} \frac{\partial}{\partial x}\left(\frac{1}{\sigma^{(s)}} \frac{\partial}{\partial x} F^{(s)}\right)+\frac{\partial^{2}}{\partial y^{2}} F^{(s)}+k^{2} F^{(s)}=0,
$$

where, and henceforth, $s=e, h$, and

$$
F^{(0)}=E_{z}^{(0)}, \quad F^{(h)}=H_{z}^{(1)}, \quad \sigma^{(0)}=\mu(x), \quad \sigma^{(l)}=\epsilon(x) .
$$

Let $G^{(s)}$ denote quantities complementary to $F^{(s)}$ such that

$$
G^{(0)}=H_{z}^{(o)}, \quad G^{(h)}=E_{z}^{(h)} .
$$

Then, (16a) and (16b) become 


$$
\frac{\partial}{\partial y} G^{(s)}=\frac{\delta^{(s)}}{\sigma^{(s)}} \frac{k_{z}}{k_{0}} \frac{\partial}{\partial x} F^{(s)}
$$

where

$$
8^{(0)}=-1, \quad 8^{(k)}=1 .
$$

The new notations introduced in (19), (20), and (22) allow the $\mathrm{E}_{\perp}$ and $\mathrm{H}_{\perp}$ fields to be treated identically in the rest of this paper.

Let a trial solution of (18) be

$$
F^{(s)}(x, y)=u^{(s)}(x) \omega^{(s)}(y) \text {. }
$$

Then the standard procedure of separation of variables leads to

$$
\sigma^{(s)} \frac{d}{d x}\left(\frac{1}{\sigma^{(s)}} \frac{d}{d x} u^{(s)}\right)+\left(k^{2}-\lambda^{(s) 2}\right) u^{(s)}=0,
$$

where $\lambda^{(s)}$ is a constant. The differential equation (24) and the boundary conditions (15) together pose a boundary-value problem which is considered in the next section.

\subsection{The Boundary-Value Problem}

In this section and the next section, for simplicity, we omit the superscript $(s)$ in the relevant quantities. Let $L$ be a differential operator defined by

$$
L=\sigma \frac{d}{d x}\left(\frac{1}{\sigma} \frac{d}{d x}\right)+k^{2} .
$$

Then the boundary-value problem for determining the eigenvalues and the eigen-functions of the modal fields is given by 


$$
\left.\begin{array}{c}
L u=\rho u, \\
u(d / 2)=e^{i \alpha_{0} d} u(-d / 2), \\
u^{\prime}(d / 2)=e^{i \varepsilon_{0} d} u^{\prime}(-d / 2),
\end{array}\right\}
$$

where a prime indicates the differentiation with respect to $x$, and

$$
p=\lambda^{2} \text {. }
$$

Let us now define an inner product $($, ) for any two integrable, complex-valued functions $u(x)$ and $v(x)$ by

$$
(u, v)=\int_{-d / 2}^{d / 2} \frac{1}{\sigma(x)} u(x) \bar{v}(x) d x,
$$

where a bar indicates the complex conjugate. For a lossless dielectric grating $(\epsilon(x)>0, \mu(x)$ $>0$ ), it is easily seen that $L$ is self-adjoint, i.e.,

$$
(L u, v)=(u, L v) \text {. }
$$

From the theory of ordinary differential equations [14], we know that the eigenvalues determined by the boundary-value problem (26) are real and they form a denumerable sequence. Furthermore, the eigen-functions form a complete, orthonormal basis in the sense that any continuous and piecewise differentiable function $f(x)$ satisfying the pseudo-periodic boundary conditions in (26) can be expanded in the eigen-functions.

In order to embrace the most general cases, however, we assume that region 0 is composed of two media of complex permittivities and permeabilities, i.e., the functions $\epsilon(x)$ and $\mu(x)$ are in general complex valued. In addition, we assume $\alpha_{0}$ and possibly $k_{z}$ to be complex. (This is a minor generalization of the works of Ref. 5-9.) This permits us to apply 
the current model to the homogeneous problem of grating diffraction [15]. In either case, the operator $L$ is no longer self-adjoint; therefore, the eigenvalues of (26) are no longer necessarily real and the eigen-functions are no longer orthogonal and complete. In order to be able to use the modal field expansions for the total field, it is necessary to consider the adjoint of (26), which is defined by

$$
\left.\begin{array}{c}
L^{*} u^{*}=\rho^{*} u^{+}, \\
u^{+}(d / 2)=e^{i \bar{k}_{0} d} u^{+}(-d / 2), \\
u^{+}(d / 2)=e^{t \bar{t}_{0} d} u^{+}(-d / 2),
\end{array}\right\}
$$

where the superscript + indicates the adjoint and $L^{+}$is the differential operator adjoint to L. It is easily seen that

$$
\left(L u, v^{+}\right)=\left(u, L^{+} v^{+}\right)
$$

if

$$
L^{+}=\bar{L}=\bar{\sigma} \frac{d}{d x}\left(\frac{1}{\bar{\sigma}} \frac{d}{d x}\right)+{\overline{K^{2}}}^{2}
$$

From the theory of non-self-adjoint boundary-value problems $[14,16]$, we know that under certain conditions, which (26) and (30) satisfy, two mutually adjoint boundary-value problems have the following properties: (a) Both boundary-value problems possess an infinite number of eigenvalues and the eigenvalues can be ordered such that

$$
\rho_{m}^{*}=\bar{\rho}_{m}, \quad m=0,1,2, \ldots
$$

(b) The eigen-functions $\left\{u_{m}\right\}$ and $\left\{u_{n}^{+}\right\}$, are bi-orthonormal, i.e.,

$$
\left(u_{m}, u_{n}^{+}\right)=\delta_{m a} \text {. }
$$

(c) Any continuous and piecewise differentiable function $f(x)$ satisfying the boundary 
conditions has a uniformly convergent formal expansion

$$
f(x)=\sum_{m=0}^{-}\left(f, u_{m}^{+}\right) u_{m}(x)
$$

Hence, even for lossy dielectric or metallic gratings, it is still mathematically justified to represent the total electromagnetic field in region 0 by a superposition of modal fields, as has been done by Botten et al.

Incidently, the Rayleigh expansions (10a,b) and (11a,b) can be viewed as expansions in basis functions

$$
e_{n}(x)=e^{i \epsilon_{n} x}
$$

It is easy to verify that $e_{n}(x)$ are eigen-functions of (26) with $L$ replaced by $d^{2} / d x^{2}$. The adjoint of this new boundary-value problem, with respect to a new inner product $\langle$, $\rangle$ defined by

$$
\langle u, v\rangle=\int_{-d / 2}^{d / 2} u(x) \bar{v}(x) d x,
$$

is (30) with $L^{+}$again replaced by $d^{2} / d x^{2}$, and

$$
e_{n}^{+}(x)=\frac{1}{d} e^{i \bar{a}_{n} x}
$$

are the adjoint eigen-functions.

\subsection{Eigenvalues and Eigen-Functions}

The explicit forms of the characteristic equation for determining the eigenvalues and 
eigen-functions can be most conveniently derived by taking advantage of the simplicity and symmetry of $\epsilon(x)$ and $\mu(x)$ given in (1). It is easy to verify that the following two functions are two linearly independent solutions of (24)

$$
\begin{gathered}
\Phi_{0}= \begin{cases}\cos \gamma_{1} x, & 0 \leq|x| \leq \frac{d_{1}}{2}, \\
\cos \frac{\gamma_{1} d_{1}}{2} \cos \gamma_{2}\left(|x|-\frac{d_{1}}{2}\right)-\frac{\sigma_{2} \gamma_{1}}{\sigma_{1} \gamma_{2}} \sin \frac{\gamma_{1} d_{1}}{2} \sin \gamma_{2}\left(|x|-\frac{d_{1}}{2}\right), & \frac{d_{1}}{2} \leq|x| \leq \frac{d}{2},\end{cases} \\
\Phi_{0}= \begin{cases}\frac{1}{\gamma_{1}} \sin \gamma_{1} x, & 0 \leq|x| \leq \frac{d_{1}}{2}, \\
\frac{1}{\gamma_{1}} \operatorname{sgn}(x)\left[\sin \frac{\gamma_{1} d_{1}}{2} \cos \gamma_{2}\left(|x|-\frac{d_{1}}{2}\right)+\frac{\sigma_{2} \gamma_{1}}{\sigma_{1} \gamma_{2}} \cos \frac{\gamma_{1} d_{1}}{2} \sin \gamma_{2}\left(|x|-\frac{d_{1}}{2}\right)\right], & \frac{d_{1}}{2} \leq|x| \leq \frac{d}{2},\end{cases}
\end{gathered}
$$

where, for $j=1,2$,

$$
\gamma_{j}^{2}=k_{j}^{2}-p
$$

Clearly, $\varphi_{e}$ is an even function and $\varphi_{0}$ is an odd function. The general solution of (24) is therefore given by

$$
u(x)=A \varphi_{0}(x)+B \varphi_{0}(x)
$$

where $A$ and $B$ are arbitrary constants. Imposition of the boundary conditions in (26) on (42) gives the characteristic equation

$$
\Delta(\rho)=\left|\begin{array}{ll}
(1-k) \varphi_{0}(d / 2) & (1+k) \varphi_{0}(d / 2) \\
(1+k) \varphi_{c}^{\prime}(d / 2) & (1-k) \varphi_{0}^{\prime}(d / 2)
\end{array}\right|=0,
$$

where

$$
K=e^{i \varepsilon_{0} d}
$$


It is obvious that when $x= \pm 1$, i.e., in Littrow mountings for which the normal incidence is a special case, $\Delta(p)$ is a product of two factors. For general angles of incidence, factorization of $\Delta(\rho)$ is impossible, and the explicit form of the characteristic equation is

$$
\cos \gamma_{1} d_{1} \cos \gamma_{2} d_{2}-\frac{1}{2}\left(\frac{\sigma_{2} \gamma_{1}}{\sigma_{1} \gamma_{2}}+\frac{\sigma_{1} \gamma_{2}}{\sigma_{2} \gamma_{1}}\right) \sin \gamma_{1} d_{1} \sin \gamma_{2} d_{2}-\cos \alpha_{0} d=0
$$

This is a transcendental equation for $\rho$, whose solutions are, in general, complex numbers.

The eigen-functions of (26), expressed in terms of $\varphi_{c}$ and $\varphi_{o}$, are given by

$$
u(x)=\left\{\begin{array}{lr}
i C \varphi_{0}(x), & \text { if } k=+1, \varphi_{0}(d / 2)=0, \\
i C \varphi_{0}(x), & \text { if } k=-1, \varphi_{0}(d / 2)=0, \\
C\left[(1+k) \varphi_{0}(d / 2) \varphi_{0}(x)-(1-k) \varphi_{0}(d / 2) \varphi_{0}(x)\right], & \text { else, }
\end{array}\right.
$$

where $C$ is the normalization constant. The eigen-functions of the adjoint problem (30) can be simply obtained by replacing the relevant quantities in (39-46) by their adjoint counterparts. It can be shown that for each eigenvalue, there is in general only one eigenfunction and accidental degeneracy of an eigenvalue can only occur in Littrow mountings. Since the normalization constants $C$ for $u(x)$ and $C^{+}$for $u^{+}(x)$ are not individually fixed by (34), we can demand $C^{+}=C$. Then it can be shown that

$$
\overline{u^{*}}(x)=x^{-1} u(-x) \text {. }
$$

This direct relationship between the two mutually adjoint eigen-functions is very useful in the numerical implementation of the theory. 


\subsection{Modal Field Representation}

Since the basis functions $\left\{u_{m}^{(s)}\right\}$ are complete and bi-orthogonal in the sense stated in 2.3 , the general solution of $(18)$ for $F^{(s)}$ can be written as

$$
F^{(s)}=\sum_{m=0}^{-} \omega_{m}^{(s)}(y) u_{m}^{(s)}(x),
$$

where

$$
\omega_{m}^{(s)}(y)=a_{m}^{(s)} \cos \lambda_{m}^{(s)} y+b_{m}^{(s)} \sin \lambda_{m}^{(s)} y,
$$

and $a_{m}{ }^{(s)}$ and $b_{m}{ }^{(s)}$ are modal field amplitudes to be determined later. Substituting (48) into (21) and integrating with respect to $y$, we obtain an expression for $G^{(s)}$,

$$
G^{(s)}=\sum_{m=0}^{\infty} x_{m}^{(s)}(y) w_{m}^{(s)}(x)
$$

where

$$
\chi_{m}^{(s)}(y)=\frac{k_{z}^{2}+\lambda_{m}^{(s) 2}}{\lambda_{m}^{(s)}}\left[-b_{m}^{(s)} \cos \lambda_{m}^{(s)} y+a_{m}^{(s)} \sin \lambda_{m}^{(s)} y\right]
$$

and

$$
w_{m}^{(s)}(x)=\frac{1}{k_{z}^{2}+\lambda_{m}^{(s) 2}} \frac{k_{2}}{k_{0}} \frac{\delta^{(s)}}{\sigma^{(s)}} \frac{d}{d x} u_{m}^{(s)}(x) .
$$

(The integration constant that would appear in (50) can be shown to be zero.) Thus, by (48), (50), and (A-1) we complete the derivation of the following modal field representation of the total electromagnetic field in region 0 :

$$
\left(\begin{array}{l}
E_{z}(x, y) \\
H_{z}(x, y)
\end{array}\right)=\sum_{m=0}^{-}\left(\begin{array}{l}
\omega_{m}^{(o)}(y) u_{m}^{(o)}(x) \\
\chi_{m}^{(o)}(y) w_{m}^{(o)}(x)
\end{array}\right)+\sum_{m=0}\left(\begin{array}{l}
\chi_{m}^{(h)}(y) w_{m}^{(h)}(x) \\
\omega_{m}^{(h)}(y) u_{m}^{(h)}(x)
\end{array}\right) .
$$


Before closing this section, we give the orthogonality relation between the vectorvalued $E_{\perp}$ solution and $H_{\perp}$ solution. For this purpose, we define an inner product [, ] of two vector-valued functions $\varphi$ and $\downarrow$ such that

$$
[\varphi, \phi]=\int_{-d / 2}^{d / 2} \varphi^{T}\left(\begin{array}{cc}
-1 / \mu & 0 \\
0 & 1 / \epsilon
\end{array}\right) \bar{\psi} d x .
$$

Let $\varphi_{m}^{(e)}=\left(u_{m}{ }^{(e)}, w_{m}^{(e)}\right)^{\mathrm{T}}$, and $\varphi_{n}^{+(h)}=\left(w_{n}^{+(h)}, u_{n}^{+(h)}\right)^{\mathrm{T}}$. Then it can be shown that

$$
\left[\varphi_{m}^{(0)}, \varphi_{n}^{+(1)}\right]=0 \text {. }
$$

\subsection{Matching Interface Conditions}

Having obtained the expressions of the electromagnetic fields in all regions of the space, we are now ready to form the final system of linear equations for determining the unknown field amplitudes by applying the interface conditions at $y= \pm h / 2$. The interface conditions are the following:

$$
\begin{array}{ll}
E_{z^{+}}=E_{z-}, & H_{z+}=H_{z-}, \\
E_{x+}=E_{x-}, & H_{x+}=H_{x-},
\end{array}
$$

where the subscripts \pm indicate limits from above and below the interface respectively, and from (9a) and (96),

$$
\begin{aligned}
& E_{x}=\frac{i}{\tilde{k}^{2}}\left(k_{z} \frac{\partial}{\partial x} E_{z}+\mu k_{0} \frac{\partial}{\partial y} H_{z}\right), \\
& H_{x}=\frac{i}{\tilde{k}^{2}}\left(k_{z} \frac{\partial}{\partial x} H_{z}-\epsilon k_{0} \frac{\partial}{\partial y} E_{z}\right) .
\end{aligned}
$$

Substituting (10a,b), (11a,b), and (53) into (56a) and (56b) and carrying out some tedious algebra, we have, for the continuity of $E_{z}$ and $H_{z}$ at $y=+h / 2$, 


$$
\begin{aligned}
& \sum_{m=0}\left[\left(\tilde{a}_{m}^{(0)}+\bar{b}_{m}^{(c)}\right) u_{m}^{(c)}(x)+\left(A_{m}^{(h)} \tilde{a}_{m}^{(h)}+B_{m}^{(h)} b_{m}^{(h)}\right) w_{m}^{(h)}(x)\right] \\
= & \tilde{I}_{z}^{(c)} e_{0}(x)+\dot{\sum}_{m=-\infty} \tilde{R}_{n}^{(o)} e_{n}(x)
\end{aligned}
$$

and

$$
\begin{aligned}
& \sum_{m=0}^{-}\left[\left(\tilde{a}_{m}^{(h)}+\tilde{b}_{m}^{(h)}\right) u_{m}^{(h)}(x)+\left(A_{m}^{(o)} \tilde{a}_{m}^{(0)}+B_{m}^{(o)} \tilde{b}_{m}^{(0)}\right) w_{m}^{(o)}(x)\right] \\
= & \tilde{I}_{z}^{(h)} e_{0}(x)+\sum_{m=-\infty}^{-} \tilde{R}_{m}^{(h)} e_{n}(x) .
\end{aligned}
$$

For the continuity of $E_{z}$ and $H_{z}$ at $y=-h / 2$, we have

$$
\sum_{m=0}^{-}\left[\left(\bar{a}_{m}^{(o)}-\tilde{b}_{m}^{(c)}\right) u_{m}^{(o)}(x)+\left(-A_{m}^{(h)} \tilde{a}_{m}^{(k)}+B_{m}^{(h)} \tilde{b}_{m}^{(l)}\right) w_{m}^{(k)}(x)\right]=\sum_{m=-\infty}^{\infty} \tilde{T}_{n}^{(o)} e_{n}(x)
$$

and

$$
\sum_{m=0}^{\infty}\left[\left(\tilde{a}_{m}^{(h)}-\sigma_{m}^{(h)}\right) u_{m}^{(h)}(x)+\left(-A_{m}^{(0)} \tilde{a}_{m}^{(0)}+B_{m}^{(0)} \tilde{b}_{m}^{(0)}\right) w_{m}^{(0)}(x)\right]=\sum_{m=-\infty}^{\infty} \tilde{T}_{n}^{(h)} e_{n}(x)
$$

For the continuity of $E_{x}$ and $H_{x}$ at $y=+h / 2$, we have

$$
\begin{aligned}
& \sum_{m=0}^{\infty}\left(A_{m}^{(0)} \tilde{a}_{m}^{(0)}+B_{m}^{(0)} b_{m}^{(o)}\right) \frac{1}{\mu} u_{m}^{(0)}(x) \\
& =\left(\tau_{1}^{(1)} \beta_{0}^{(1)} \tilde{I}_{z}^{(0)}+\tau_{3}^{(1)} \alpha_{0} \tilde{I}_{z}^{(h)}\right) e_{0}(x)-\sum_{n=-\infty}^{\infty}\left(\tau_{1}^{(1)} \beta_{n}^{(1)} \tilde{R}_{n}^{(0)}-\tau_{3}^{(1)} \alpha_{n} \tilde{R}_{n}^{(n)}\right) e_{n}(x)
\end{aligned}
$$

and

$$
\begin{aligned}
& \sum_{m=0}^{\infty}\left(A_{m}^{(1)} \tilde{a}_{m}^{(h)}+B_{m}^{(h)} \tilde{b}_{m}^{(k)}\right) \frac{1}{\epsilon} u_{m}^{(n)}(x) \\
= & \left(\tau_{2}^{(1)} \beta_{0}^{(1)} \tilde{I}_{2}^{(n)}-\tau_{3}^{(1)} \alpha_{0} \tilde{I}_{2}^{(c)}\right) e_{0}(x)-\sum_{n=-\infty}^{-}\left[\tau_{2}^{(1)} \beta_{n}^{(1)} \tilde{R}_{n}^{(h)}+\tau_{3}^{(1)} \alpha_{n} \tilde{R}_{n}^{(c)}\right] e_{n}(x) .
\end{aligned}
$$

Finally, for the continuity of $E_{x}$ and $H_{x}$ at $y=-h / 2$, we have 


$$
\sum_{m=0}^{\infty}\left(-A_{m}^{(0)} \tilde{d}_{m}^{(0)}+B_{m}^{(0)} \dot{b}_{m}^{(0)}\right) \frac{1}{\mu} u_{m}^{(0)}(x)=\sum_{n=-\infty}^{\infty}\left(\tau_{1}^{(2)} \beta_{n}^{(2)} \tilde{T}_{n}^{(0)}+\tau_{3}^{(2)} \alpha_{n} \tilde{T}_{n}^{(k)}\right) e_{n}(x)
$$

and

$$
\sum_{m=0}^{\infty}\left(-A_{m}^{(h)} \tilde{a}_{m}^{(n)}+B_{m}^{(h)} \tilde{b}_{m}^{(h)}\right) \frac{1}{\epsilon} u_{m}^{(h)}(x)=\sum_{n=-\infty}^{\infty}\left(\tau_{2}^{(2)} \beta_{n}^{(2)} \tilde{T}_{n}^{(h)}-\tau_{3}^{(2)} \alpha_{n} \tilde{T}_{n}^{(\epsilon)}\right) e_{n}(x)
$$

In the above equations the unknowns are

$$
\begin{array}{cc}
\tilde{a}_{m}^{(s)}=a_{m}^{(s)} \cos \left(\lambda_{m}^{(s)} h / 2\right), & \tilde{b}_{m}^{(s)}=b_{m}^{(s)} \sin \left(\lambda_{m}^{(s)} h / 2\right), \\
\tilde{R}_{n}^{(s)}=R_{n}^{(s)} e^{i \beta_{n}^{(1)} h / 2}, \quad \tilde{T}_{n}^{(s)}=T_{n}^{(s)} e^{i \beta_{m}^{(s)} k / 2},
\end{array}
$$

and the rest of the new notations are defined in Appendix B. The numerical solution of equations (58) and (59) will be considered in section 3.2 .

\subsection{Diffraction Efficiency and Polarization}

Once $\bar{R}_{n}^{(s)}$ and $\bar{T}_{n}^{(s)}$ are solved from (58) and (59), $R_{n}^{(s)}$ and $T_{n}^{(s)}$ are given by (61). By virtue of (9a) and (9b), all quantities of practical interest can be readily expressed in terms of $R_{n}^{(s)}$ and $T_{n}^{(s)}$. Suppose the media in regions 1 and 2 are lossless, and the incident plane wave is normalized such that

$$
\frac{\beta_{0}^{(1)}}{E^{(1) 2}}\left(\epsilon^{(1)}\left|I_{z}^{(o)}\right|^{2}+\mu^{(1)}\left|I_{z}^{(k)}\right|^{2}\right)=1
$$

Then, the diffraction efficiencies for the reflected and the transmitted propagating waves of order $n$ are given by 


$$
\eta_{n}^{(1)}=\frac{\beta_{n}^{(1)}}{\xi^{(1) 2}}\left(\epsilon^{(1)}\left|R_{n}^{(0)}\right|^{2}+\mu^{(1)}\left|R_{n}^{(h)}\right|^{2}\right)
$$

and

$$
\eta_{n}^{(2)}=\frac{\beta_{n}^{(2)}}{\bar{k}^{(2) 2}}\left(\epsilon^{(2)}\left|T_{n}^{(0)}\right|^{2}+\mu^{(2)}\left|T_{n}^{(n)}\right|^{2}\right) .
$$

If the media in region 0 are also lossless, the energy balance theorem holds:

$$
\sum_{n} \eta_{n}^{(1)}+\sum_{n^{\prime}} \eta_{n^{\prime}}^{(2)}=1
$$

where $n$ and $n$ ' run through all propagating orders in regions 1 and 2 , respectively.

In many applications involving conical mountings, it is very important to be able to predict the states of polarization of the diffracted orders. Let us associate with a propagating order having a wave-vector

$$
k_{n}^{(n)}=\dot{x} \alpha_{n} \pm \hat{y} \beta_{n}^{(n)}+z k_{2} \text {, }
$$

two unit vectors $S_{n}^{())}$and $\hat{p}_{n}^{(0)}$ such that

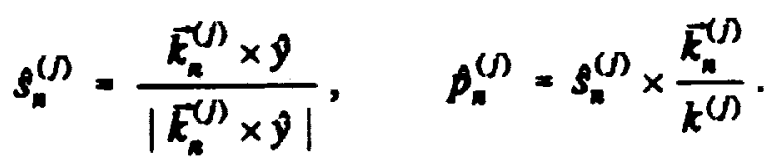

In (65) the plus sign is for $j=1$, and the minus sign is for $j=2$. The polarization of this diffracted order can be described with the following two angular parameters [17]: 


$$
\left.\begin{array}{l}
\alpha_{n}^{(j)}=\arctan \left(\frac{\left|E_{m}^{(j)}\right|}{\left|E_{n \varphi}^{(j)}\right|}\right), \quad 0 \leq \alpha_{n}^{(j)} \leq \frac{\pi}{2}, \\
\delta_{n}^{(j)}=-\arg \left(\frac{E_{m}^{(j)}}{E_{\pi p}^{(j)}}\right), \quad-\pi<\delta_{n}^{(j)} \leq \pi,
\end{array}\right\}
$$

where $E_{n s}^{(j)}$ and $E_{n p}{ }^{(j)}$ are the $s$ - and $p$-components of the electric field. (The notation $\alpha_{n}^{(j)}$ should not be confused with $\alpha_{n}$ defined in (12).) It is a simple exercise to show that for a diffracted wave in medium 1 ,

$$
\frac{E_{n o}^{(1)}}{E_{n}^{(1)}}=\sqrt{\frac{\mu^{(1)}}{\epsilon^{(1)}}} \frac{\alpha_{n} \epsilon^{(1)} k_{0} R_{n}^{(0)}+\beta_{n}^{(1)} k_{z} R_{n}^{(b)}}{\alpha_{n} \mu^{(1)} k_{0} R_{n}^{(b)}-\beta_{n}^{(1)} k_{z} R_{n}^{(b)}} .
$$

The expression for a diffracted wave in medium 2 may be obtained from (68) by replacing $R_{n}^{(s)}$ by $T_{n}^{(s)}$, superscript (1) by (2), and $\beta_{n}^{(1)}$ by $-\beta_{n}^{(2)}$.

\section{NUMERICAL ASPECTS}

\subsection{Solution of the Characteristic Equation}

An efficient, reliable, and accurate numerical method for solving the characteristic equation (45) is of vital importance to the performance of a computer program implementing the modal analysis. Two very different numerical methods have been employed. The method of Botten et al. $[7,18]$ is a general one capable of finding all zeros of an analytic function in a prescribed region of the complex plane. That of Suratteau et al.

[8] and Tayeb and Petit [9] is a problem-specific method that takes advantage of the fact that (45) can be factored in Littrow mountings. Both of these methods systematically find all eigenvalues of (45) in a prescribed region of the complex plane, and both of them 
perform well even for highly conducting gratings. As reported by the authors of the second method, their method is as effective as the first method but requires significantly shorter computation time. For this reason, we have decided to use the second method in our numerical implementation of this work. For details of these numerical methods, the interested reader is referred to the references cited above.

\subsection{Solution of the Field Amplitudes}

Before embarking on the numerical solution of (58) and (59), we first analyze the composition of these equations. In (58) and (59) there are eight equations and eight sets of unknowns. The right-hand sides of these equations are expanded in basis functions $\left\{e_{n}\right\}$, which are bi-orthogonal to their adjoint $\left\{e_{n}^{+}\right\}$. The left-hand sides are expanded in four different sets of functions $\left\{u_{m}^{(e)}\right\},\left\{u_{m}^{(h)}\right\},\left\{w_{m}^{(e)}\right\}$, and $\left\{w_{m}{ }^{(h)}\right\}$. Of these four, as scalarvalued functions, $\left\{u_{m}{ }^{(e)}\right\}$ and $\left\{u_{m}{ }^{(h)}\right\}$ are bi-orthogonal to their respective adjoints, but not to each other. The functions $\left\{w_{m}{ }^{(e)}\right\}$ and $\left\{w_{m}{ }^{(h)}\right\}$ are proportional to the derivatives of $\left\{u_{m}{ }^{(e)}\right\}$ and $\left\{u_{m}{ }^{(h)}\right\}$ and they are not orthogonal to any other functions.

Equations (58) and (59) constitute a system of equations in known function expansions with unknown expansion coefficients. Such a system can be solved by the method of moments [19], which consists of three steps. First, a projection basis, i.e., a set of linearly independent testing functions, is chosen. Then, both sides of the series expansion equations are projected onto this basis by forming appropriate inner products with the testing functions. This step eliminates the $x$-dependence of the equations and produces an 
algebraic linear system of equations of infinite dimension. Finally, the linear system is truncated to a finite order and its solution is obtained by the standard numerical techniques.

Since equations (58) and (59) are already expanded in terms of bi-orthonormal basis functions, it is advantageous to choose the adjoints of these basis functions as the testing functions so that the subsequent numerical solution can be simplified. For each of the eight equations we have two convenient projection bases, $\left\{u_{m}^{+(s)}\right\}$ and $\left\{e_{n}^{+}\right\}$. Therefore, there can be many different combinations of choices of projection bases for the overall system. Following Suratteau et al., a projection method in which the interface conditions for the $z$ components are projected onto one basis and those for the $x$-components are projected onto the other basis is called a hybrid method. A projection method in which all interface conditions are projected onto one basis is called a homogeneous method. For the case of non-conical mountings, Suratteau et al. have proved that the numerical solutions resulting from the hybrid methods satisfy the energy balance and reciprocity criteria automatically (independent of the truncation orders) while those resulting from the homogeneous methods do not. It can be shown that the above statement is also true in the case of conical mountings.

In this paper, we adopt the homogeneous method utilizing the projection basis $\left\{u_{m}^{+(s)}\right\}$, so that the energy balance and reciprocity criteria are not automatically satisfied. Multiplying equations (58) by $a_{m}^{+(s)}(x) / \sigma^{(s)}(x)$, and equations (59) by $a_{m}^{+(s)}(x)$, then integrating over a grating period, and making use of the bi-orthogonality relation (34), we have the 
following matrix equations:

$$
\begin{aligned}
& W Y=U X+U I, \\
& D Y=Q X+P I,
\end{aligned}
$$

where

$$
X=\left(\begin{array}{c}
\tilde{R}^{(o)} \\
\tilde{T}^{(0)} \\
\tilde{R}^{(h)} \\
\tilde{T}^{(h)}
\end{array}\right), \quad Y=\left(\begin{array}{c}
\tilde{a}^{(o)} \\
\tilde{b}^{(0)} \\
\tilde{a}^{(l)} \\
\tilde{b}^{(0)}
\end{array}\right), \quad I=\left(\begin{array}{c}
\tilde{I}_{z}^{(o)} \delta_{0 n} \\
0 \\
\tilde{I}_{z}^{(h)} \delta_{o n} \\
0
\end{array}\right),
$$

$\delta_{0 n}$ is the Kronecker delta, and the rest of the matrices are defined in Appendix B. In (71). each element of the column vectors is itself a column vector, and the elements of $X$ and $Y$ are related to the unknown field amplitudes listed in (60) and (61). The matrix $D$ in (70) is diagonal, so vector $Y$ can be expressed in terms of $X$ without numerical matrix inversion. Substituting the expression of $Y$ into (69), we have

$$
\left(W D^{-1} Q-U\right) X=\left(U-W D^{-1} P\right) I .
$$

This is the final linear system of equations from which we numerically determine the field amplitudes.

In order to solve the linear system (72) on a computer, we unavoidably have to truncate the matrices. We designate $N$ as the total number of terms retained in Rayleigh expansions (we truncate the Rayleigh expansions symmetrically with respect to the zero diffraction order) and $M$ as that retained in the modal expansions. The integers $N$ and.$M$ are called the truncation orders. It is easily seen that for the solution of (72) to be well 
(neither under- nor over-) specified, the two truncation orders must be the same. Thus, the matrices $W, U, P, Q$, and $D$ in (72) are $4 N$ by $4 N$ square matrices.

\section{NUMERICAL EXAMPLES}

In this section we present some numerical results. The computer program is written in Fortran 77 and double precision is used for real and complex arithmetic. For the special case of non-conical mountings, the program has been checked using published data with good agreement. For the general case, it meets the energy balance and reciprocity criteria with reasonable accuracy. Table 1 tabulates TE and TM diffraction efficiencies of a metallic grating in a non-conical mounting. The data of Botten et al. are taken from Table 1 of Ref. 6. Note that Botten et al adopted a hybrid projection method that allows unequal truncation orders $N$ and $M$. Clearly, the agreement is very good, especially for the TE polarization.

To date, there is no numerical data, especially data of the polarization parameters, for non-perfectly conducting lamellar gratings in conical mountings available in the literature; therefore, we present some original data in the rest of this section. Listed in Table 2 are diffraction efficiencies $(\eta)$, polarization angles $(\alpha, \delta)$, and diffraction angles $(\theta, \phi)$ of a dielectric grating in a conical mounting (the parameters are listed in the table caption). Note that the incident plane wave is right-hand, circularly polarized. Listed in Table 3 are diffraction efficiencies $(\eta)$, polarization angles $(\alpha, \delta)$, and diffraction angles $(\theta, \phi)$ of a highly conducting grating in a conical mounting. The incident plane wave is linearly polarized with 
equal $s$ and $p$ component amplitudes. In both cases, the truncation orders are chosen to ensure that the accuracy of the data is better than one percent (see discussion below).

In Figs. $3 a, 3 b$ and $3 c$ we show the change of diffraction efficiencies $\eta$ and polarization angles $\alpha$ and $\delta$ of a small period dielectric grating as the incident azimuthal angle $\phi$ sweeps through the first quadrant. The incident plane wave is always $p$ polarized and it strikes the grating from the optically denser medium at a polar angle greater than the critical angle (for total internal reflection). This configuration is reminiscent of what occurs in a planar waveguide grating coupler for a TM polarized guided-wave [20]. The reflected and the transmitted negative first orders pass off at about $\phi=37^{\circ}$ and $\phi=60^{\circ}$, respectively. It is evident that as soon as $\phi$ is nonzero, the diffraction orders become elliptically polarized. The two first orders are nearly circularly polarized at $\phi-20^{\circ}$. Also, the senses of polarization of the two first orders remain right-handed throughout the angular range of their existence.

Next, we illustrate the excellent convergence rate of the modal method. For this purpose, we define a measure of error $\Delta_{N}$ as follows

$$
\Delta_{N}=\log _{10}\left|\frac{f_{N}-f^{*}}{f^{*}}\right|,
$$

where $f_{N}$ stands for any one of the physical quantities, such as a diffraction efficiency or a polarization angle, computed with truncation order $N$, and $f=f_{N^{*}}$, where $N^{*}>N$ is an integer. If $f$ stands for the sum of the diffraction efficiencies for a lossless grating, $f=1.0$. 
Roughly speaking, the negative of $\Delta_{N}$ gives the number of correct significant digits in the numerical results.

Fig. 4 shows the convergence of the sum of the diffraction efficiencies and the efficiencies of the negative first orders in reflection and in transmission for a dielectric grating. The physical parameters are the same as those of Table 2. The truncation order $N$ varies from 11 to 61 in increment of 2 , and $N^{*}=63$. It is evident from the figure that better than one percent accuracy is achieved as soon as the truncation order is greater than 11. The convergence is not monotonic. The large oscillation in the convergence sequence of the reflected order is probably due to the smallness of the diffaction efficiency (see row 3, column 2 of Table 2). If we make a low order polynomial fit of each set of the data in the figure, the three resulting curves will have more or less similar shapes and close locations. This implies that the energy balance criterion can be used as a good accuracy indicator, thanks to our choice of the homogeneous projection method.

Fig. 5 shows the convergence of the diffraction efficiency and diffraction angles $\alpha$ and $\delta$ of the negative first order of a metallic grating. The physical parameters are the same as those of Table 3. The truncation order $\mathrm{N}$ varies from 11 to 69 in increment of 2 , and $N^{*}=$ 71. Since now the grating is metallic, the convergence in this case is, as expected, slower than that of Fig. 4. However, better than one percent accuracy can still be achieved with a truncation orders of 40 or greater. 
As the authors of Ref. 5-8 have shown, one of the unique features of the modal method is that it works very well even for deep, highly conducting gratings. This feature is also true for the extension of the method to conical mountings as developed in this paper. This is understandable, since the fundamental analytical and numerical issues for conical and non-conical mountings are essentially the same. For the sake of saving space, however, we will not provide any numerical evidence here.

\section{SUMMARY}

In this paper, we have extended the rigorous modal method of Botten et al. to the case of conical mountings. A crucial step in accomplishing the extension is the field decomposition discussed in section 2.2 and Appendix $\mathrm{A}$. The field decomposition reduces the vector-valued boundary-value problem given by (14) and (15) to a scalar one given by (26), thus tremendously simplifying the subsequent analysis and allowing the previous works of Botten et al. and Suratteau et al be used here. The completeness and orthogonality of the modal fields in the corrugated region are carefully established. The computer program implementing the extended modal method can treat a plane wave of arbitrary angle of incidence and polarization. It converges very well for highly conducting grating materials and very deep grating grooves. We have included some original numerical data of both diffraction efficiencies and diffraction polarizations for conical diffraction configurations.

The mathematical formulation presented in this paper has been kept general. In fact, the explicit $x$-dependence of $\epsilon(x)$ and $\mu(x)$ given in (1) is not used, except for the derivation 
of the characteristic equation and the eigen-functions in section 2.4. Therefore, the formulation is valid for any periodic $\epsilon(x)$ and $\mu(x)$. However, it is precisely the simple $x$ dependence of $\epsilon(x)$ and $\mu(x)$ in (1) that makes the easy derivations in section 2.4 possible. For any other permittivity and permeability variations, the solutions for the eigenvalues and eigen-functions become very complicated, and the modal analysis quickly loses its advantages over other grating methods.

The present analysis can be easily extended to treat gratings of arbitrary groove shapes and waveguide gratings with a number of uniform layers above and below the corrugated region. These extensions will be the subject of a future paper.

\section{ACKNOWLEDGMENT}

This work is supported by the Optical Data Storage Center at the University of Arizona.

\section{APPENDICES}

\subsection{Appendix A}

Theorem:

Any solution of (14) and (15) can be decomposed such that

$$
\left(\begin{array}{l}
E_{z} \\
H_{z}
\end{array}\right)=\left(\begin{array}{l}
E_{z}^{(o)} \\
H_{z}^{(o)}
\end{array}\right)+\left(\begin{array}{l}
E_{z}^{(h)} \\
H_{z}^{(h)}
\end{array}\right),
$$

where $\left(E_{z}^{(e)}, H_{z}^{(e)}\right)^{\mathrm{T}}$ and $\left(E_{z}^{(h)}, H_{z}^{(h)}\right)^{\mathrm{T}}$ are $E_{\perp}$ and $H_{\perp}$, respectively, and they satisfy (14) 
and (15) independently. The decomposition (A-1) is, in general, unique.

Proof:

Since there is no explicit $y$-dependence in (14), any solution of (14) is necessarily of the following form

$$
\left(\begin{array}{l}
E_{z} \\
H_{z}
\end{array}\right)=\sum_{\lambda}\left(\begin{array}{l}
E_{2 \lambda}(x, y) \\
E_{2 \lambda}(x, y)
\end{array}\right),
$$

where

$$
\left(\begin{array}{l}
E_{2 \lambda}(x, y) \\
H_{2 \lambda}(x, y)
\end{array}\right)=e^{i \lambda y}\left(\begin{array}{l}
\xi_{\lambda}(x) \\
\eta_{\lambda}(x)
\end{array}\right) .
$$

Substituting (A-3) into (14), and eliminating the $y$-dependence, we have

$$
\left(\begin{array}{lr}
\frac{k^{2}}{\epsilon} \frac{d}{d x}\left(\frac{\epsilon}{k^{2}} \frac{d}{d x}\right)+\tilde{k}^{2}-\lambda^{2} & +i \lambda \frac{k_{z}}{k_{0}} \frac{k^{2}}{\epsilon}\left(\frac{d}{d x} \frac{1}{k^{2}}\right) \\
-i \lambda \frac{k_{z}}{k_{0}} \frac{k^{2}}{\mu}\left(\frac{d}{d x} \frac{1}{k^{2}}\right) & \frac{k^{2}}{\mu} \frac{d}{d x}\left(\frac{\mu}{k^{2}} \frac{d}{d x}\right)+k^{2}-\lambda^{2}
\end{array}\right)\left(\begin{array}{l}
\xi_{\lambda}(x) \\
\eta_{\lambda}(x)
\end{array}\right)=0 .
$$

Equation (A-4) is a linear, two-dimensional vector-valued, second-order ordinary differential equation. It, therefore, has four linearly independent solutions. Suppose $\left(E_{z \lambda}, H_{z \lambda}\right)^{\mathrm{T}}$ is $\mathrm{E}$. Then, from (16a)

$$
\eta_{\lambda}(x)=-\frac{1}{i \lambda} \frac{1}{\mu} \frac{k_{z}}{k_{0}} \frac{d}{d x} \xi_{\lambda}(x)
$$

Substitution of (A-5) into (A-4) results in two second-order equations for $\xi_{2}(x)$. These two equations are not independent; one can be derived from the other. Thus, we have two linearly independent solutions of (A-4) that satisfy the $E_{\perp}$ condition (16a). Similarly, if we demand $\left(E_{z \lambda}, H_{z \lambda}\right)^{\mathrm{T}}$ to be $\mathrm{H}_{\perp}$, then 


$$
\xi_{\lambda}(x)=\frac{1}{i \lambda} \frac{1}{\epsilon} \frac{k_{z}}{k_{0}} \frac{d}{d x} \eta_{2}(x)
$$

and we have two linearly independent solutions of (A-4) that satisfy the $\mathrm{H}_{\perp}$ condition (16b). Clearly, the solutions of $E_{\perp}$ type and $\mathrm{H}_{\perp}$ type are linearly independent. Therefore, any solution of (A-4) has the following form

$$
\left(\begin{array}{l}
\xi_{\lambda}(x) \\
\eta_{\lambda}(x)
\end{array}\right)=c_{\lambda 1}^{(o)}\left(\begin{array}{l}
u_{\lambda 1}^{(o)} \\
v_{\lambda 1}^{(o)}
\end{array}\right)+c_{\lambda 2}^{(o)}\left(\begin{array}{l}
u_{\lambda 2}^{(o)} \\
v_{\lambda 2}^{(o)}
\end{array}\right)+c_{\lambda 1}^{(h)}\left(\begin{array}{c}
v_{\lambda 1}^{(h)} \\
u_{\lambda 1}^{(h)}
\end{array}\right)+c_{\lambda 2}^{(h)}\left(\begin{array}{l}
v_{\lambda 2}^{(h)} \\
u_{\lambda 2}^{(h)}
\end{array}\right),
$$

where $c_{\mu}{ }^{(s)}, l=1,2, s=e, h$, are constants, $\left(u_{\mu}{ }^{(e)}, v_{\mu}{ }^{(e)}\right)^{\mathrm{T}}$ and $\left(v_{\mu}(h), u_{\mu}{ }^{(h)}\right)^{\mathrm{T}}$ are $\mathrm{E}_{\perp}$ and $\mathrm{H}_{\perp}$ solutions of (A-4) respectively. So, the decomposition (A-1) is always possible.

Next, we impose the pseudo-periodic boundary conditions (15) on the general solution (A-7). This leads to the following characteristic equation

$$
\left|\begin{array}{llll}
U_{\lambda 1}^{(o)} & U_{\lambda 2}^{(o)} & V_{\lambda 1}^{(h)} & V_{\lambda 2}^{(h)} \\
U_{\lambda 1}^{()^{\prime}} & U_{\lambda 2}^{()^{\prime}} & V_{\lambda 1}^{\left(h^{\prime}\right.} & V_{\lambda 2}^{()^{\prime}} \\
V_{\lambda 1}^{(0)} & V_{\lambda 2}^{(0)} & U_{\lambda 1}^{(h)} & U_{\lambda 2}^{(h)} \\
V_{\lambda 1}^{(c)} & V_{\lambda 2}^{(o)^{\prime}} & U_{\lambda 1}^{(h)^{\prime}} & U_{\lambda 2}^{(h)^{\prime}}
\end{array}\right|=0,
$$

where

$$
\begin{aligned}
& U_{\lambda l}^{(s)}=u_{\lambda l}^{(s)}(d / 2)-e^{i a_{0} d} u_{\lambda l}^{(s)}(-d / 2), \\
& v_{\lambda l}^{(s)}=v_{\lambda l}^{(s)}(d / 2)-e^{l e_{0} d} v_{\lambda l}^{(s)}(-d / 2) \text {, } \\
& U_{\lambda l}^{(s)}=u_{\lambda l}^{(s)}(d / 2)-e^{i a_{0} d} u_{\lambda l}^{(s)^{\prime}}(-d / 2) \text {, } \\
& V_{\lambda l}^{(s)^{\prime}}=v_{\lambda l}^{(s)^{\prime}}(d / 2)-e^{i e_{0} d} v_{\lambda l}^{(s)^{\prime}}(-d / 2) \text {. }
\end{aligned}
$$

By elementary row manipulation and making use of (A-5) and (A-6) for the $E_{\perp}$ and $H_{\perp}$ 
solutions, it can be shown that the two off-diagonal two-by-two matrices in (A-8) can be made zero. Therefore, the $E_{\perp}$ and $H_{\perp}$ solutions of (A-4), and hence those of (14), satisfy the boundary conditions (15) independently.

Suppose the decomposition (A-1) is not unique. Then we may have a decomposition of the zero field into two non-zero orthogonal modal fields. Furthermore, each of these fields satisfies both the $E_{\perp}$ and the $H_{\perp}$ conditions simultaneously. However, this leads to $k_{z}^{2}+\lambda^{2}=0$, a condition which is, in general, not true. This completes the proof of the theorem.

\subsection{Appendix $B$}

As in the main text of the paper, $j=1,2$, and $s=e, h$. In (58) and (59)

$$
\begin{gathered}
\tilde{I}_{z}^{(s)}=I_{z}^{(s)} e^{-i \beta_{0}^{(j) h / 2} .} \\
A_{m}^{(s)}=\frac{k_{z}^{2}+\lambda_{m}^{(s) 2}}{\lambda_{m}^{(s)}} \tan \frac{\lambda_{m}^{(s)} h}{2}, \quad B_{m}^{(s)}=\frac{k_{z}^{2}+\lambda_{m}^{(s) 2}}{\lambda_{m}^{(s)}} \operatorname{ctan} \frac{\lambda_{m}^{(s)} h}{2}, \\
\tau_{1}^{(j)}=i k_{0}^{2} \frac{\epsilon^{(j)}}{\tilde{k}^{(j) 2}}, \quad \tau_{2}^{(n)}=i k_{0}^{2} \frac{\mu^{(j)}}{\tilde{k}^{(j) 2}}, \quad \tau_{3}^{(j)}=i k_{0} \frac{k_{z}}{\tilde{k}^{(j) 2}} .
\end{gathered}
$$

For the sake of clarity, the matrices in (69) and (70) are expressed in block forms, followed by the definitions of each sub-matrix.

$$
W=\left(\begin{array}{cccc}
1 & 1 & W^{(o)} A^{(h)} & W^{(0)} B^{(h)} \\
1 & -1 & -W^{(0)} A^{(h)} & W^{(c)} B^{(h)} \\
W^{(h)} A^{(o)} & W^{(h)} B^{(o)} & 1 & 1 \\
-W^{(h)} A^{(o)} & W^{(h)} B^{(s)} & 1 & -1
\end{array}\right) .
$$




$$
\begin{aligned}
& U=\left(\begin{array}{cccc}
U^{(0)} & 0 & 0 & 0 \\
0 & U^{(0)} & 0 & 0 \\
0 & 0 & U^{(h)} & 0 \\
0 & 0 & 0 & U^{(h)}
\end{array}\right) . \\
& D=2\left(\begin{array}{cccc}
A^{(o)} & 0 & 0 & 0 \\
0 & B^{(c)} & 0 & 0 \\
0 & 0 & A^{(h)} & 0 \\
0 & 0 & 0 & B^{(h)}
\end{array}\right) \\
& Q=\left(\begin{array}{cccc}
\tilde{U}^{(c)} & 0 & 0 & 0 \\
0 & \tilde{U}^{(0)} & 0 & 0 \\
0 & 0 & \tilde{U}^{(1)} & 0 \\
0 & 0 & 0 & \tilde{U}^{(1)}
\end{array}\right)\left(\begin{array}{cccc}
-\tau_{1}^{(1)} \beta^{(1)} & -\tau_{1}^{(2)} \beta^{(2)} & \tau_{3}^{(1)} \alpha & -\tau_{3}^{(2)} \alpha \\
-\tau_{1}^{(1)} \beta^{(1)} & \tau_{1}^{(2)} \beta^{(2)} & \tau_{3}^{(1)} \alpha & \tau_{3}^{(2)} \alpha \\
-\tau_{3}^{(1)} \alpha & \tau_{3}^{(2)} \alpha & -\tau_{2}^{(1)} \beta^{(1)} & -\tau_{2}^{(2)} \beta^{(2)} \\
-\tau_{3}^{(1)} \alpha & -\tau_{3}^{(2)} \alpha & -\tau_{2}^{(1)} \beta^{(1)} & \tau_{2}^{(2)} \beta^{(2)}
\end{array}\right) . \\
& A_{m}^{(s)}=A_{m}^{(s)} \delta_{m n}, \quad B_{m a}^{(s)}=B_{m}^{(s)} \delta_{m a} . \\
& \alpha_{m n}=\alpha_{n} \delta_{m i n}, \quad \beta_{m n}^{(J)}=\beta_{n}^{(J)} \delta_{m n} . \\
& U_{m}^{(s)}=\left(e_{n}, u_{m}^{+(s)}\right)_{s}, \quad \tilde{U}_{m}^{(s)}=\left\langle e_{n}, u_{m}^{+(s)}\right\rangle . \\
& W_{m=1}^{(c)}=\left(w_{n}^{(h)}, u_{m}^{+(e)}\right)_{e}, \quad W_{m}^{(h)}=\left(w_{n}^{(o)}, u_{m}^{+(h)}\right)_{h} .
\end{aligned}
$$

To get the expression for matrix $P$, we only need to reverse the sign of $\beta^{())}$in (B-7). In (B10) and (B-11) the inner products are those defined in (28) and (37), and the subscript $s$ indicates that the weight function $\sigma^{(s)}$ should be used in the integral. By using (55) and (47), it can be shown that the two sub-matrices in (B-11) are related such that

$$
W_{m i n}^{(0)}+W_{m i n}^{(h)}=0
$$

Hence, there are five sub-matrices involving the eigen-functions to be calculated. It can be 
shown that these sub-matrix elements can be expressed in terms of the left and right limits of $u_{m}^{(s)}$ and its derivative at $\pm d_{1} / 2$, if the periodic medium is characterized by (1).

Since $w_{m}^{(s)}$ and $r_{3}^{())}$are proportional to $k_{r}$, the matrices defined in (B-4) through (B7) become block-diagonalized when $k_{z}=0$. Of course, this means that in non-conical mountings, $E_{z}$ and $\mathrm{H}_{2}$ are de-coupled.

\section{REFERENCES}

1. R. E. Collin, "Reflection and Transmission at a Slotted Dielectric Interface," Canadian J. Phys., 34(4), 398-411 (1956).

2. C. B. Burckhardt, "Diffraction of a Plane Wave at a Sinusoidally Stratified Dielectric Grating," J. Opt. Soc. Am., 56(11), 1502-1509 (1966).

3. K. Knop, "Rigorous Diffraction Theory for Transmission Phase Gratings with Deep Rectangular Grooves," J. Opt. Soc. Am., 68(9), 1206-1210 (1978).

4. S. T. Peng, T. Tamir, and H. L. Bertoni, "Theory of Periodic Dielectric Waveguides," IEEE Trans. Microw. Theory \& Tech., 23(1), 123-133 (1975).

5. L. C. Botten, M. S. Craig, R. C. McPhedran, J. L. Adams, and J. R. Andrewarthat. "The Dielectric Lamellar Diffraction Grating," Optica Acta, 28(3), 413-428 (1981).

6. L. C. Botten, M. S. Craig, R. C. McPhedran, J. L. Adams, and J. R. Andrewartha. "The Finitely Conducting Lamellar Diffraction Grating," Optica Acta, 28(8), 1087-11112 (1981).

7. L. C. Botten, M. S. Craig, and R. C. McPhedran, "Highly Conducting Lamellar 
Diffraction Gratings," Optica Acta, 28(8), 1103-1106 (1981).

8. J. Y. Suratteau, M. Cadilhac, and R. Petit, "Sur la Détermination Numérique des Efficacités de Certains Réseaux Diélectriques Profonds," J. Optics (Paris), 14(6), 273288 (1983).

9. G. Tayeb and R. Petit, "On the Numerical Study of Deep Conducting Lamellar Diffraction Gratings," Optica Acta, 31(12), 1361-1365 (1984)

10. P. Sheng, R. S. Stepleman, and P. N. Sanda, "Exact Eigenfunctions for Square-Wave Gratings: Application to Diffraction and Surface-Plasmon Calculations," Phys. Rev., B26(6), 2907-2916 (1982).

11. C. W. Haggans, L. Li, T. Fujita, and R. K. Kostuk, "Lamellar Gratings as Polarization Components for Specularly Reflected Beams," submitted to the special issue on diffractive optics, J. Mod. Opt.

12. S. T. Peng, "Rigorous Formulation of Scattering and Guidance by Dielectric Grating Waveguides: General Case of Oblique Incidence," J. Opt. Soc. Am., A6(12), 1869-1883 (1989).

13. R. Petit, "A Tutorial Introduction," in Electromagnetic Theory of Gratings, ed. R. Petit (Springer-Verlag, Berlin, 1980).

14. R. H. Cole, Theory of Ordinary Differential Equations (Appleton-Century-Crofts, New York, 1968).

15. M. Neviere, "The Homogeneous Problem," in Electromagnetic Theory of Gratings, ed. R. Petit (Springer-Verlag, Berlin, 1980).

16. M. A. Naimark, Linear Differential Operators, Part I (Frederick Ungar, New York, 
1967).

17. See, for example, M. Born and E. Wolf, Principles of Optics, 6th ed. (Pergamon Press, Oxford, 1989).

18. L. C. Botten, M. S. Craig, and R. C. McPhedran, "Complex Zeros of Analytic Functions," Computer Physics Communications, 29, 245 (1983).

19. R. F. Harrington, Field Computation by Moment Methods (Macmillan, New York, 1968).

20. L. Li, Q. Gong, G. N. Lawrence, and J. J. Burke, "Polarization Properties of Planar Dielectric Waveguide Gratings," Apph Opt, to be published. 


\section{TABLE CAPTIONS}

1. Numerical comparison with the results of Botten et al for a metallic grating in a nonconical mounting. Parameters: $d=1.0 \mu \mathrm{m}, d_{1}=0.4001 \mu \mathrm{m}, h=0.1 \mu \mathrm{m}, \epsilon^{(1)}=\epsilon^{(2)}$ $=\epsilon_{1}=1.0, \epsilon_{2}=(1.5+i 1.0)^{2}, \lambda_{0}=0.8 \mu \mathrm{m}, \theta=11.5^{\circ}, \phi=0^{\circ}$. Truncation orders: Botten et al, $M=20, N=51$; this paper, $M=N=31$.

2. Diffraction efficiencies ( $\eta$, polarization angles $(\alpha, \delta)$, and diffraction angles $(\theta, \phi)$ of a dielectric grating in a conical mounting. All angular values are in degrees. Parameters: $d=1.0 \mu \mathrm{m}, d_{1}=0.5 \mu \mathrm{m}, h=0.5 \mu \mathrm{m}, \epsilon^{(1)}=\epsilon_{1}=1.0, \epsilon^{(2)}=\epsilon_{2}=2.25$, $\lambda_{0}=0.5 \mu \mathrm{m}$. Incident polarization: $\alpha=45^{\circ}, \delta=90^{\circ}$. Incident angle: $\theta=\phi=45^{\circ}$. Truncation orders: $M=N=31$.

3. Diffraction efficiencies $(\eta)$, polarization angles $(\alpha, \delta)$, and diffraction angles $(\theta, \phi)$ of a metallic grating in a conical mounting. All angular values are in degrees. Parameters: $d=1.0 \mu \mathrm{m}, d_{1}=0.5 \mu \mathrm{m}, h=1.0 \mu \mathrm{m}, \epsilon^{(1)}=\epsilon_{1}=1.0, \epsilon^{(2)}=\epsilon_{2}=$ $(0.1+i 5.0)^{2}, \lambda_{0}=0.5 \mu \mathrm{m}$. Incident polarization: $\alpha=45^{\circ}, \delta=0^{\circ}$. Incident angle: $\theta=30^{\circ}, \phi=45^{\circ}$. Truncation orders: $M=N=51$. 


\section{FIGURE CAPTIONS}

1. The coordinate system for a lamellar grating in a conical mounting.

2. The geometry of a lamellar grating.

3. Diffraction efficiencies $\eta$ (Fig. $a$ ) and polarization angles $\alpha$ (Fig. $b$ ) and $\delta$ (Fig. $c$ ) of a dielectric grating versus the incident azimuthal angle $\phi$. The polarization angles are measured in degrees. Parameters: $d=0.3 \mu \mathrm{m}, d_{1}=0.15 \mu \mathrm{m}, h=0.15 \mu \mathrm{m}, \epsilon^{(1)}=$ $\epsilon_{1}=1.5, \epsilon^{(2)}=\epsilon_{2}=1.0, \lambda_{0}=0.5 \mu \mathrm{m}$. Incident polarization: $\alpha=0^{\circ}$. Incident polar angle: $\theta=60^{\circ}$. Truncation orders: $M=N=45$.

4. Illustration of convergence for a dielectric grating. Round dots: sum of all diffraction efficiencies. Hollow squares: diffraction efficiency of - 1 order in reflection. Hollow triangles: diffraction efficiency of - 1 order in transmission. The parameters are the same as for Table 2.

5. Illustration of convergence for a metallic grating. Round dots: diffraction efficiency of -1 order. Hollow squares: polarization angle $\alpha$. Hollow triangles: polarization angle $\delta$. The parameters are the same as for Table 3 . 


\begin{tabular}{|c|c|c|c|c|c|c|c|}
\hline \multirow{2}{*}{ 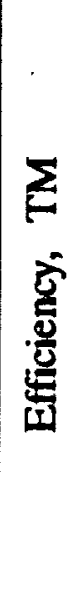 } & 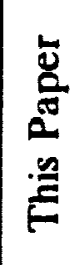 & 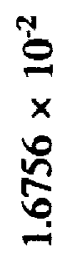 & 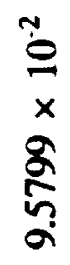 & $\begin{array}{l}\frac{3}{x} \\
\frac{5}{5} \\
\frac{n}{y}\end{array}$ & $\begin{array}{l}\tilde{\theta} \\
x \\
\dot{x} \\
\stackrel{\mathscr{P}}{ \pm} \\
\underset{j}{\sim}\end{array}$ & 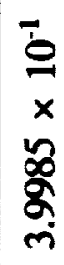 & $\begin{array}{l}\vec{\beta} \\
x \\
\infty \\
\tilde{n} \\
\ddot{n}\end{array}$ \\
\hline & 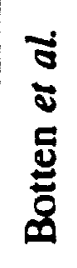 & 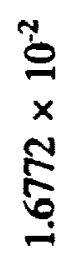 & $\begin{array}{l}\tilde{\sigma} \\
x \\
\tilde{n} \\
\tilde{n} \\
\end{array}$ & $\begin{array}{l}\vec{g} \\
x \\
\hat{x} \\
\frac{8}{y}\end{array}$ & 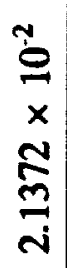 & $\begin{array}{l}\dot{\delta} \\
\dot{x} \\
\dot{\hat{g}} \\
\dot{\delta}\end{array}$ & 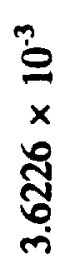 \\
\hline \multirow{2}{*}{ 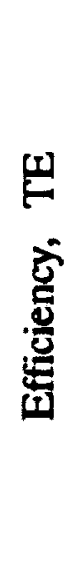 } & 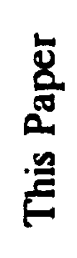 & 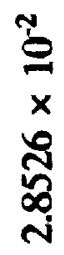 & 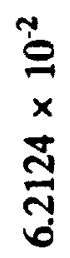 & 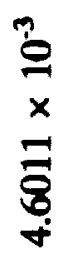 & $\begin{array}{l}\underset{b}{\infty} \\
x \\
\frac{\tilde{m}}{5} \\
\infty \\
\dot{m}\end{array}$ & $\begin{array}{l}\overrightarrow{\dot{O}} \\
\dot{x} \\
\tilde{g} \\
\dot{g}\end{array}$ & $\begin{array}{l}\stackrel{3}{0} \\
x \\
2 \\
8 \\
\& \\
\dot{8}\end{array}$ \\
\hline & 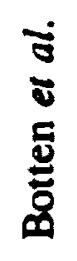 & 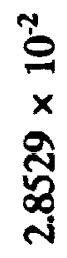 & 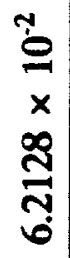 & 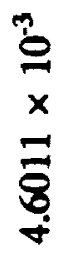 & $\begin{array}{l}\dot{b} \\
\dot{x} \\
\dot{\mathbb{Z}} \\
\infty \\
\dot{m} \\
\dot{m}\end{array}$ & $\begin{array}{l}\overrightarrow{0} \\
x \\
\dot{a} \\
\dot{\sigma} \\
\dot{\sigma}\end{array}$ & $\begin{array}{l}\hat{0} \\
\dot{x} \\
\dot{8} \\
\dot{8} \\
\dot{8}\end{array}$ \\
\hline & 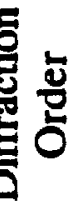 & $\bar{\alpha}$ & 2 & $\vec{\alpha}$ & $F^{\top}$ & $\Leftrightarrow$ & $F$ \\
\hline
\end{tabular}




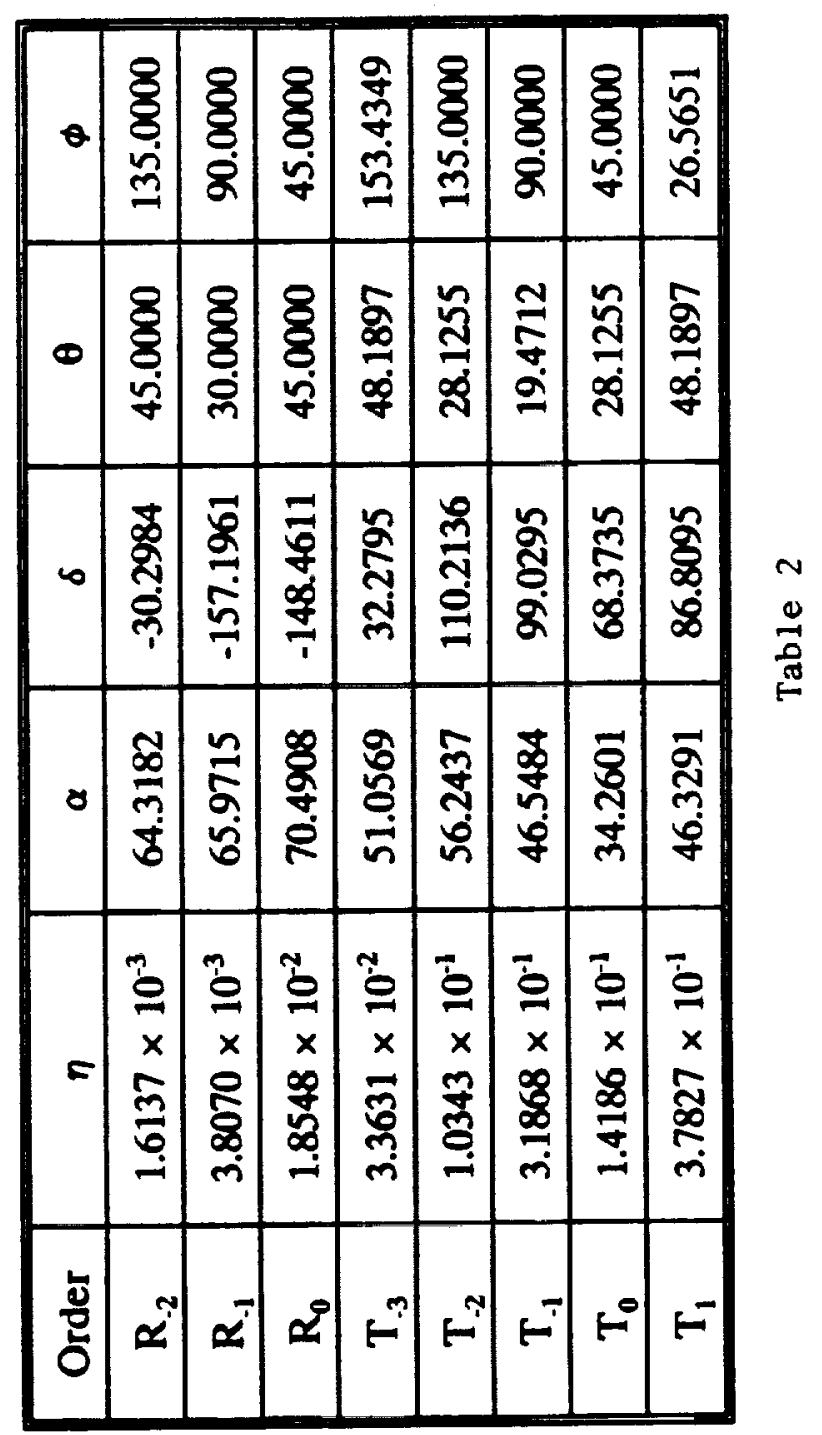




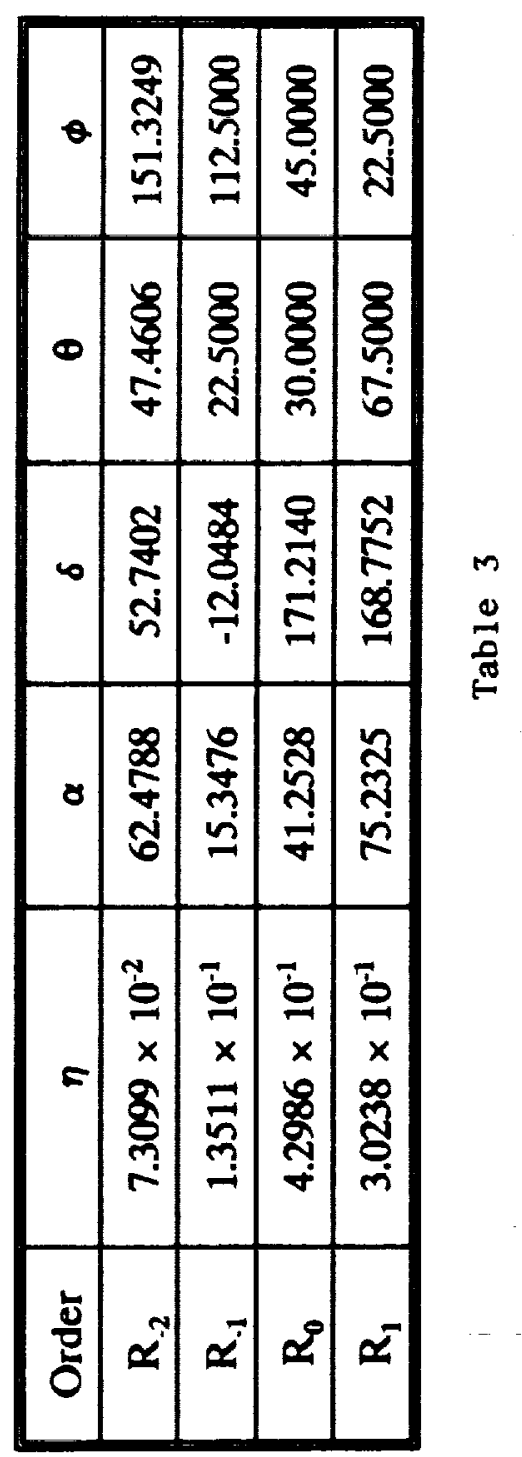




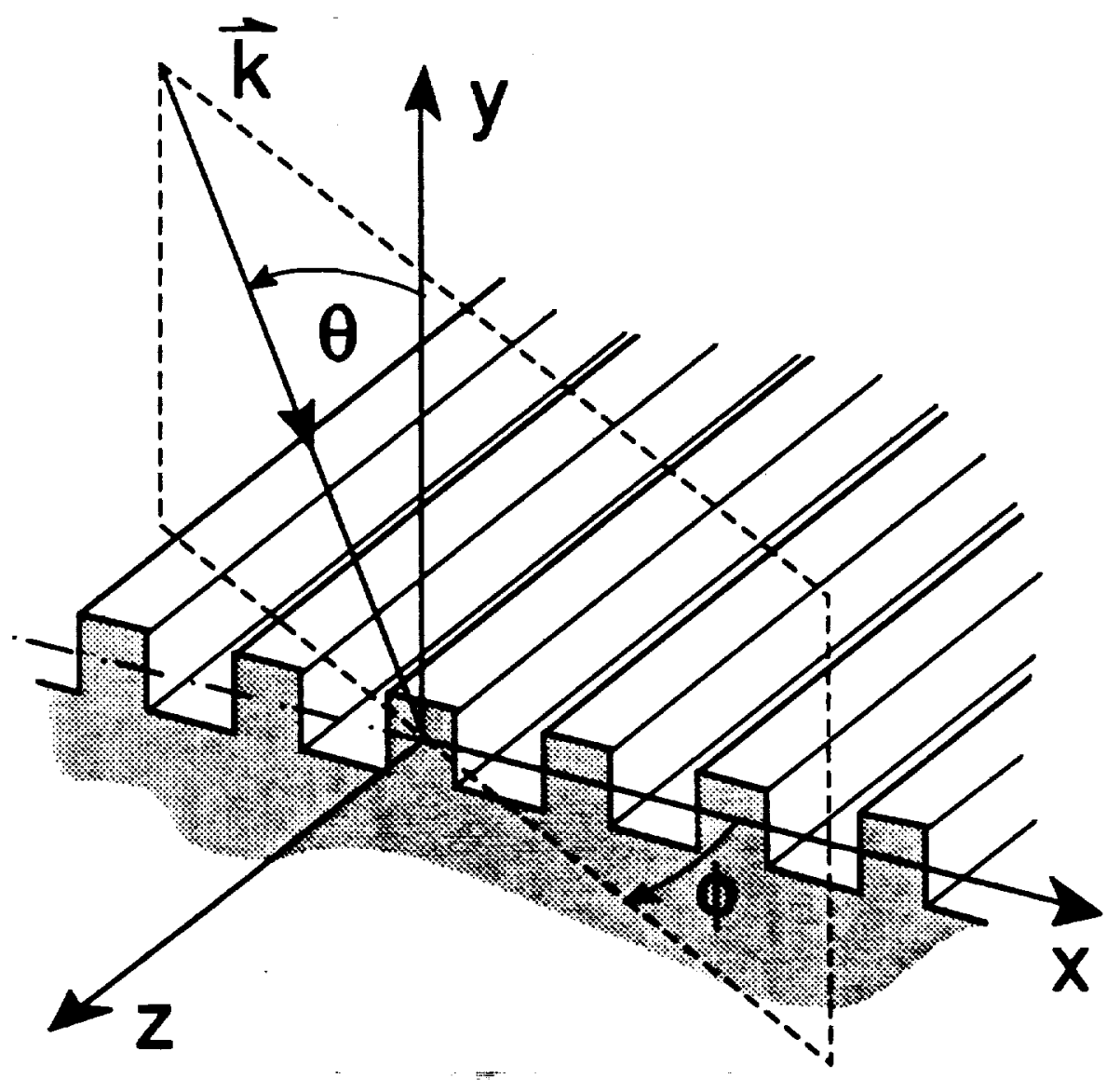

Fig. 1 


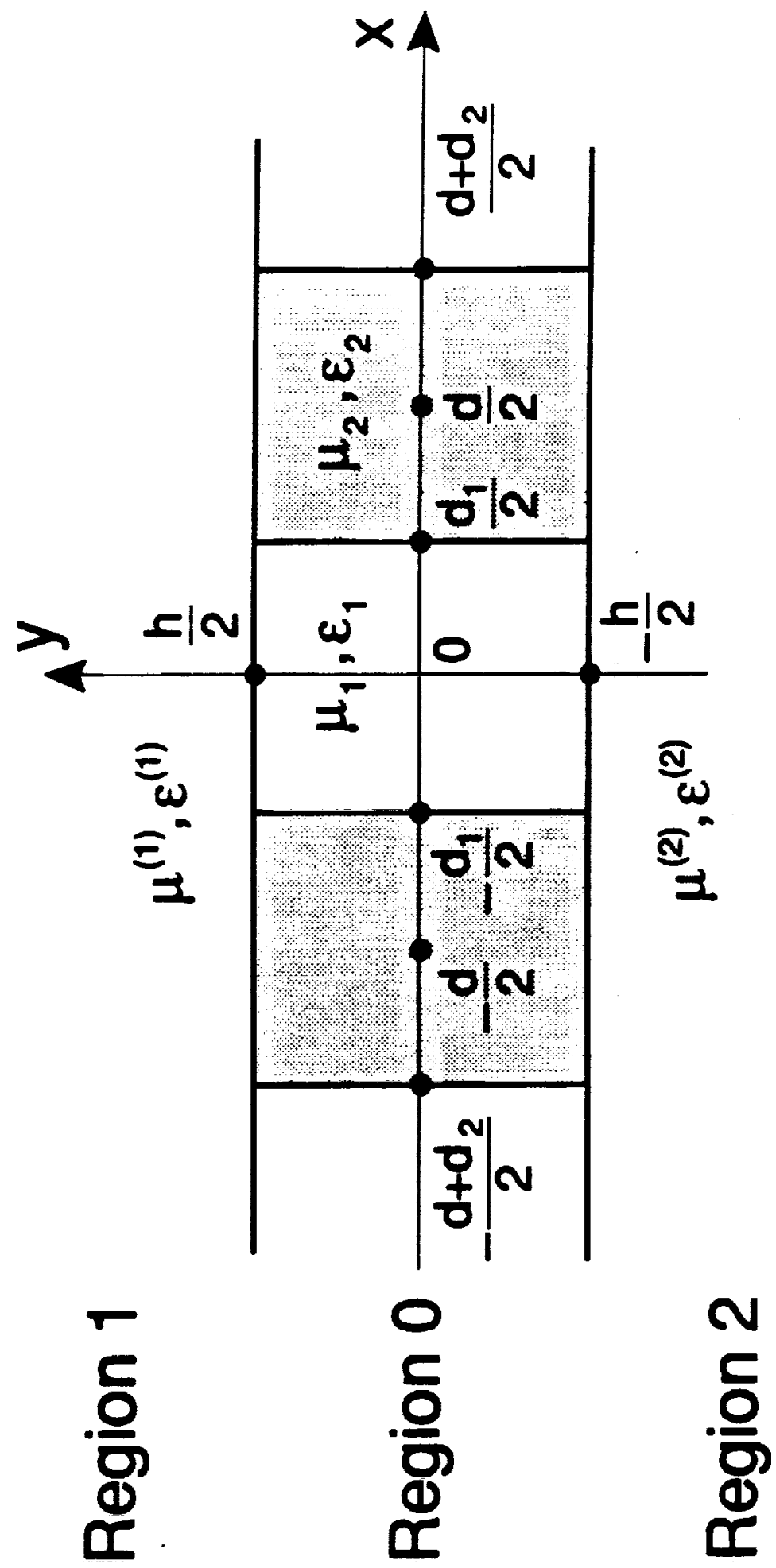




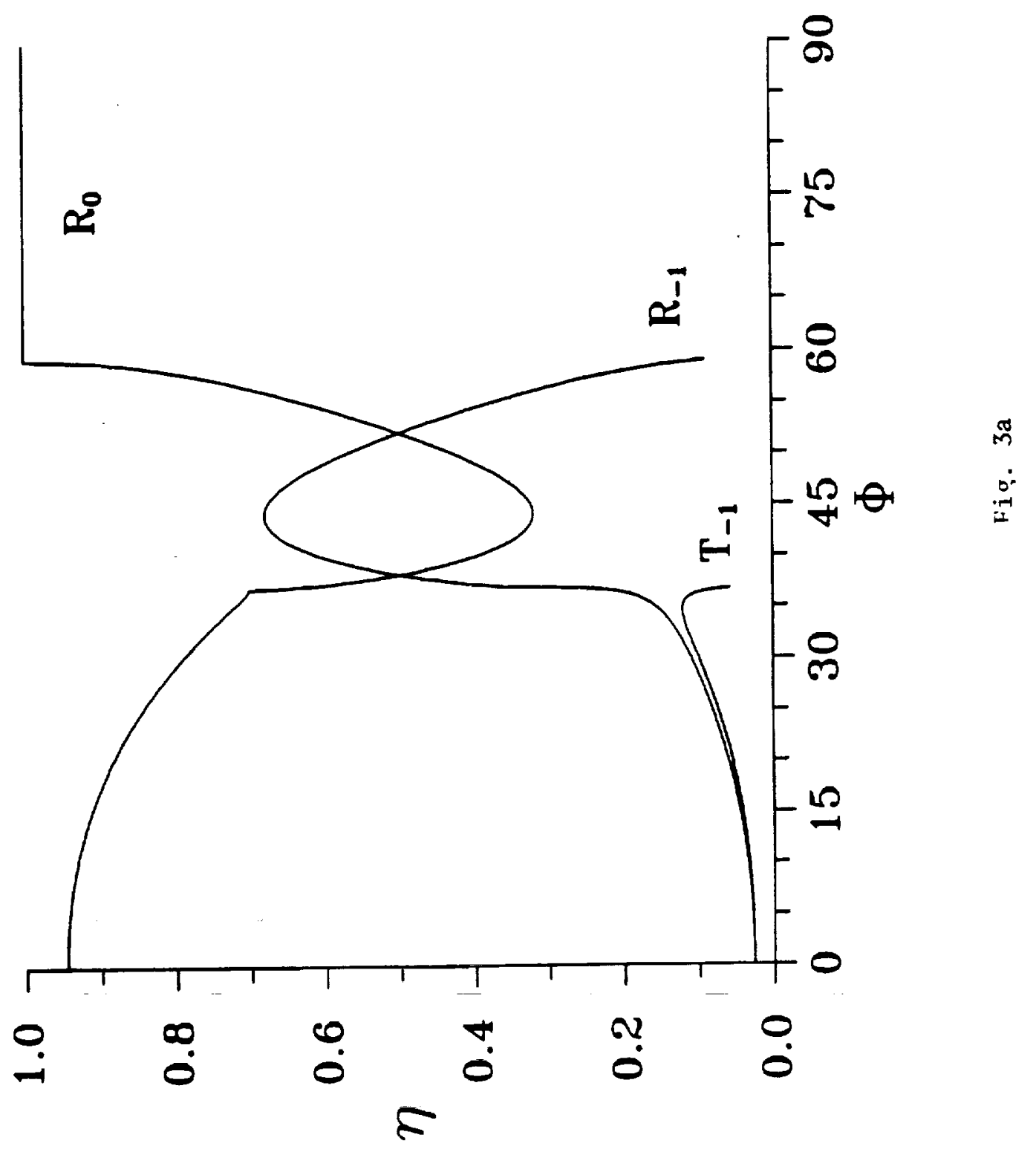




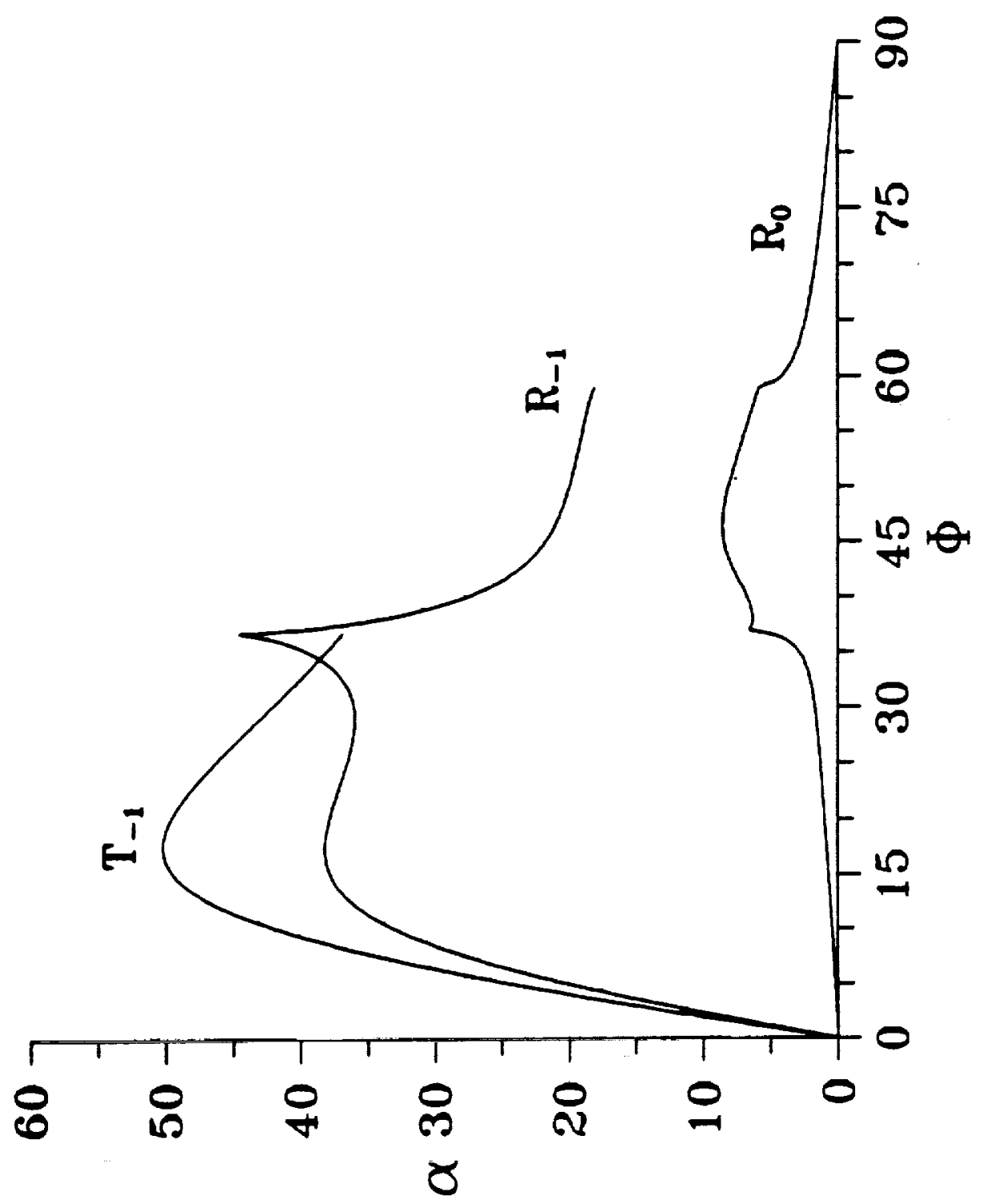

ले
$\dot{a}$
$\dot{1}$ 


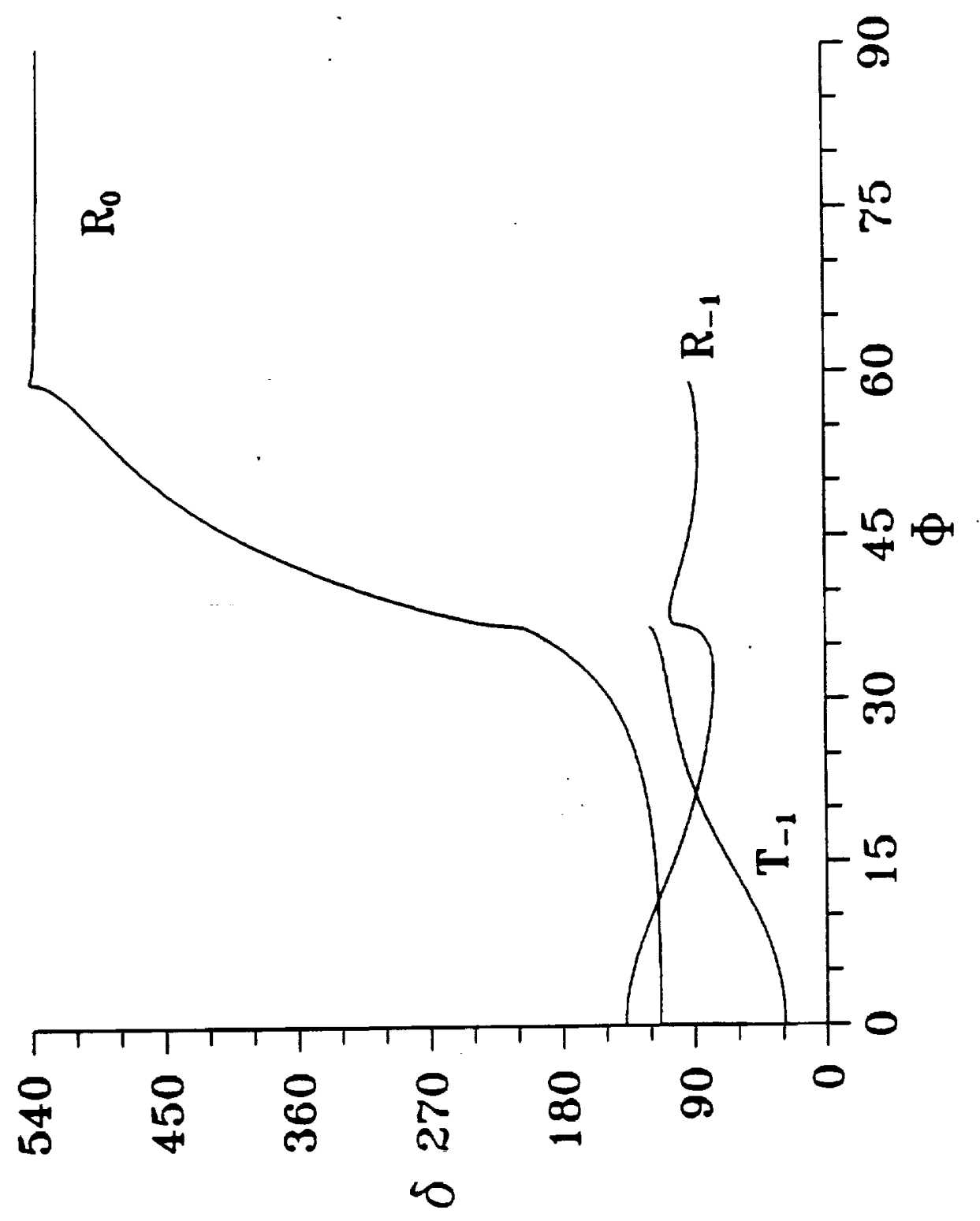

m 


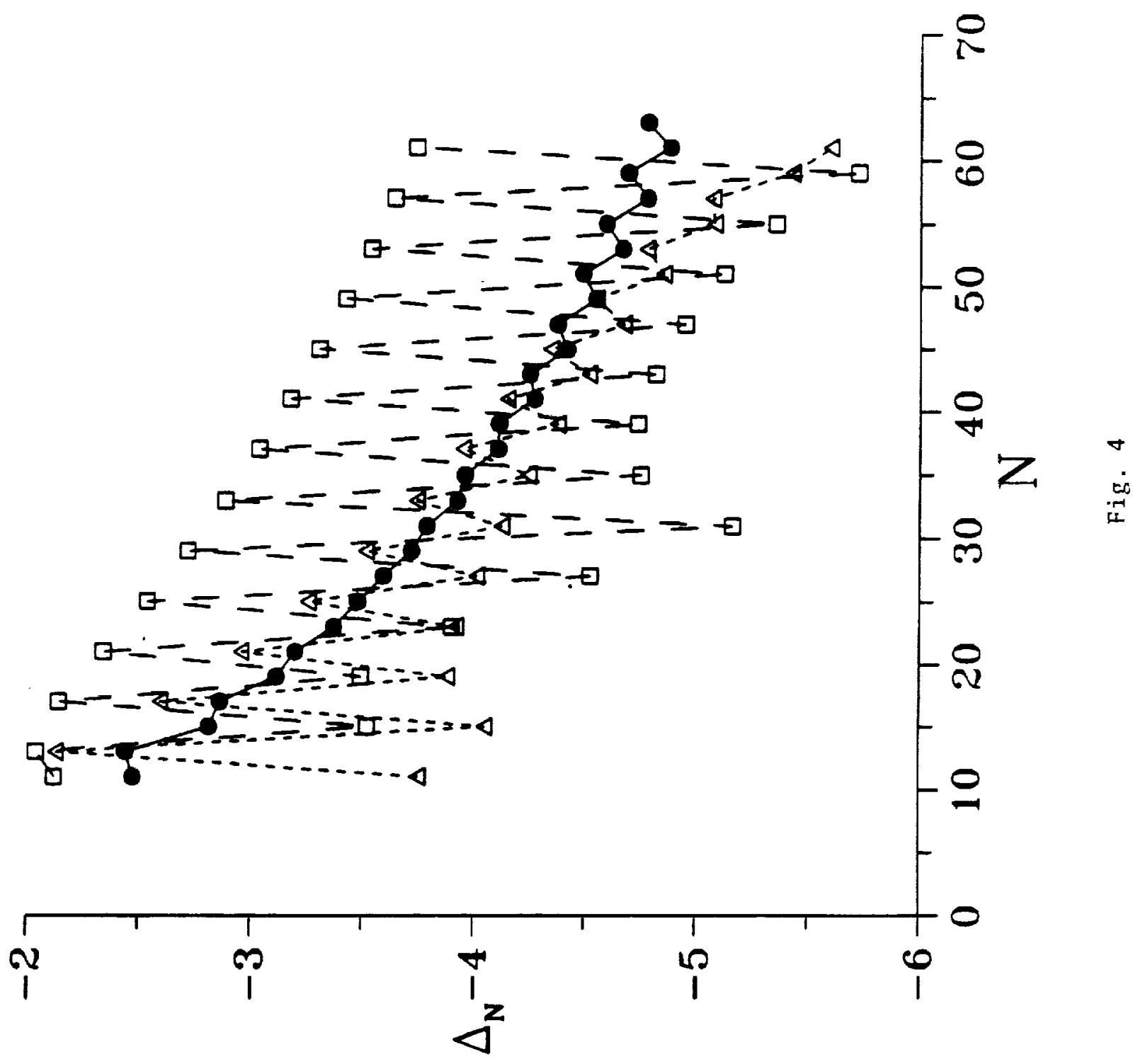




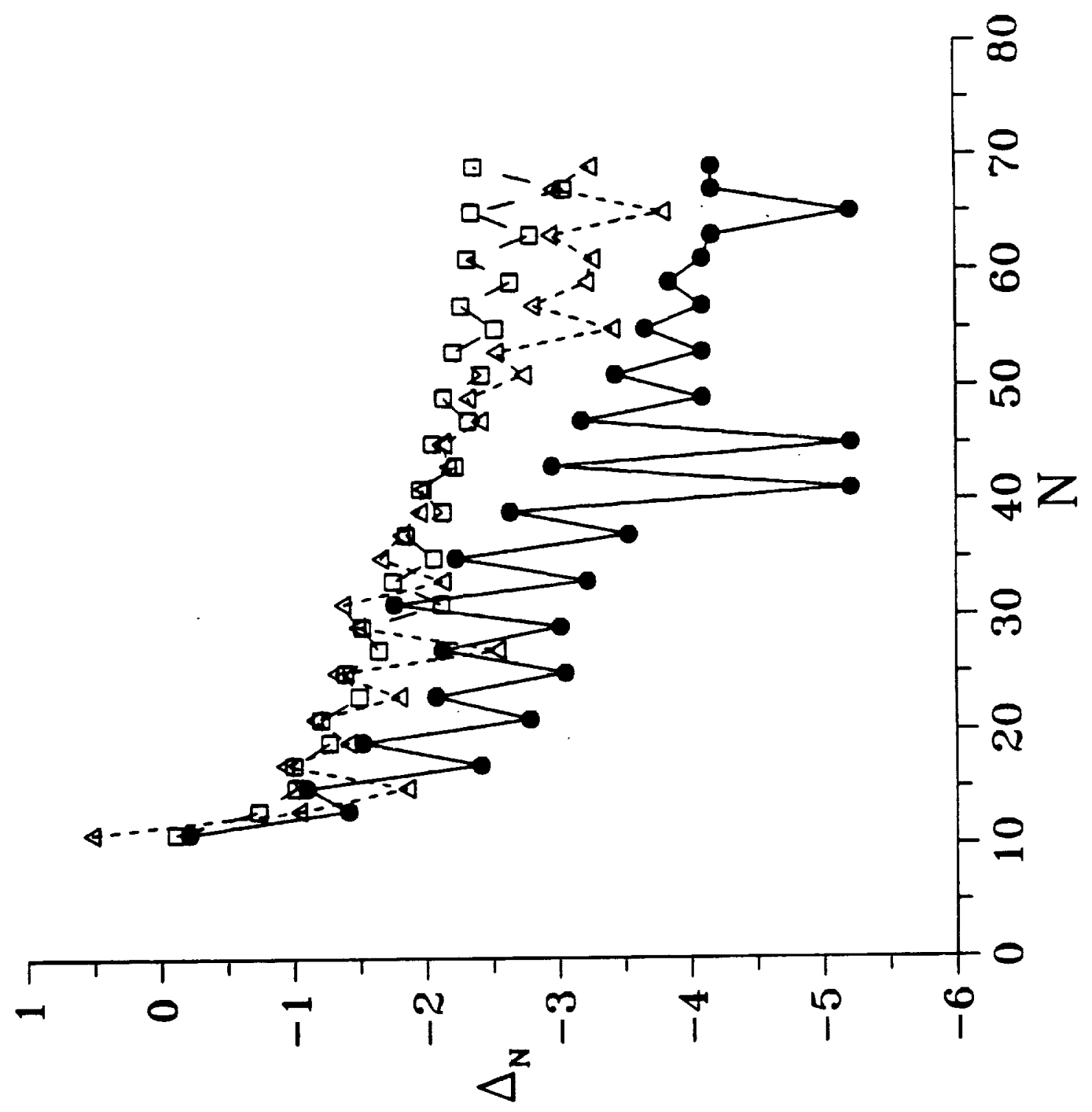


$\omega$

$\sigma$

$\nabla$

$\sigma$

$\sigma$

-

$-$ 


\section{APPENDIX 0}


-

$=$

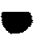

$\sigma$

0

$\sigma$ 